\title{
Development and validation of time-series synthesizers of rain attenuation for Ka-band and $Q / V$-band satellite communication systems
}

\author{
Joël Lemorton ${ }^{1, *, \dagger}$, Laurent Castanet $^{1}$, Frédéric Lacoste ${ }^{2}$, Carlo Riva ${ }^{3}$, Emilio \\ Matricciani $^{3}$, Uwe-Carsten Fiebig ${ }^{4}$, Max Van de Kamp ${ }^{5,}$ and Antonio Martellucci ${ }^{6}$ \\ ${ }^{1}$ ONERA-ElectroMagnetics \& Radar Department, 2 Avenue E. Belin-BP 4025, 31055 Toulouse Cedex 4, France \\ ${ }^{2}$ CNES, 18 Avenue Edouard Belin, 31401 Toulouse Cedex 4, France \\ ${ }^{3}$ Dipartimento di Ellettronica e Informazione, Politecnico di Milano, 20133 Milano, Italy \\ ${ }^{4}$ Institute for Communications and Navigation, German Aerospace Research (DLR), D-82230 Wessling, Germany \\ ${ }^{5}$ University of Bath, Bath BA2 7 AY, U.K. \\ ${ }^{6}$ ESA/ESTEC, TOS-EEP, P.O. Box 299, Noordwijk 2200-AG, The Netherlands
}

\begin{abstract}
SUMMARY
The aim of this paper is to present recent developments in terms of propagation time-series synthesizers, carried out in the framework of the ESA study $16865 / 03 / \mathrm{NL} / \mathrm{EC}$ 'Development of propagation models for telecommunication satellite systems' (ONERA Final Report RF 4/07757/DEMR, 2004). The paper is composed of three parts: a review of the initial requirements related to propagation time series for system performance simulation, a description of a collection of rain attenuation time-series synthesizers and of their related input parameters, and a comparative analysis of the output characteristics of these time-series synthesizers as compared with experimental data collected during the OLYMPUS and ITALSAT propagation experiments. Copyright (C) 2007 John Wiley \& Sons, Ltd.
\end{abstract}

Received 20 July 2006; Revised 27 June 2007; Accepted 2 July 2007

KEY WORDS: propagation modelling; time-series synthesizer; time-series generator; attenuation; rain; scintillation; OLYMPUS; ITALSAT

\footnotetext{
*Correspondence to: Joël Lemorton, ONERA—ElectroMagnetics \& Radar Department, 2 Avenue E. Belin—BP 74025, 31055 Toulouse Cedex 4, France.

† E-mail: Joel.Lemorton@onera.fr

\$Previously with ONERA.

Contract/grant sponsor: ESA External Fellowship
} 


\section{INTRODUCTION}

Propagation modelling has gained great importance in the design of satellite telecom systems above $10 \mathrm{GHz}$. In Ka-band (20-30 GHz), $Q / V$-band $(40-50 \mathrm{GHz})$ and above the transmitted signal can be attenuated severely by precipitation, clouds and other meteorological effects [1]. With the trend to use higher frequency bands, attenuation can become very high and, thus, has a great impact on the system performance, since signal attenuation degrades the received carrierto-noise ratio $C / N_{0}$. If the received $C / N_{0}$ falls below the target $C / N$, the communication link does not fulfil the quality of service requirements, i.e. the link is not available. Low or reduced link availability, however, is often not acceptable for service providers and their customers. As a consequence, the compensation of signal attenuation is a very important task.

Fade compensation is done by the so-called fade mitigation techniques (FMTs). A great variety of FMTs exists: static straightforward fade margin implementation as well as dynamic countermeasures such as adaptive modulation and coding, adaptive data rate switching and adaptive spot beam antennas [2]. Today the most widely applied FMT is the implementation of a fade margin, which compensates signal attenuation as long as it does not exceed the implemented fade margin. The application of a fade margin does not require any interaction between transmitter and receiver and is therefore the preferred choice in many satellite systems, especially below Ku-band. On the other hand, a large fade margin is not always feasible, especially above Ku-band, and moreover could be a waste of resources, since the communication link would be substantially over designed under clear sky conditions. Furthermore, over-designed links often cause interference problems.

A considerably better approach in terms of resource optimization is the implementation of adaptive FMTs. The design and optimization of adaptive FMTs, however, have to be carried out very carefully and require refined information on the propagation channel, i.e. not only statistical distribution of yearly attenuation but also information on the dynamics of attenuation and scintillation. Computer simulations can significantly help the optimization of FMTs [3]. Computer simulations in turn require an appropriate channel model for fading which is most suitably provided by a time-series generator, able to provide time samples of the attenuated signal with the same behaviour as 'real-world' time-series.

The development of time-series generators of rain fading is a recent and still ongoing research topic for fixed satellite systems, although it had been already considered before for mobile systems in urban environment. First approaches were made in 1999-2000 [4-6]. In 2001, the COST activity COST 280 'Propagation Impairment Mitigation for Millimeter Wave Radio Systems' was started with the goal to promote research on channel models and adaptive FMTs. A preliminary review of various channel model approaches has been presented at COST 280 [7].

The aim of this paper is to present recent developments in terms of propagation time-series synthesizers. In the first part, the initial requirements related to propagation time series and to the dynamics of the propagation channel for system performance simulation are listed, focussing on the requested inputs and on the expected outputs of time-series synthesizers. In the second part, the basic principles of the time-series synthesizers considered in this study are presented; they are essentially stochastic and database-oriented time-series synthesizers. In the third part, some results of comparisons with respect to experimental data collected during the OLYMPUS and ITALSAT propagation experiments are given, dealing with the long-term behaviour of the channel. The aim is to compare the performances of different time-series synthesizers and to have a preliminary insight about their respective validation domains. 
A methodology dealing with the short-term behaviour has also been developed and tested but will not be presented here.

\section{SYSTEM REQUIREMENTS}

After a brief overview of today's satellite multimedia communication systems, and after a description of propagation issues relevant for the design and optimization of FMTs, the system requirements for time-series synthesizers are presented.

\subsection{Context of satellite multimedia communication systems}

The current state of the telecommunication market is driven by the increasing demand of the end users for multimedia services, which require high data rates. Within the fixed satellite service, frequency bandwidths wide enough to carry such high data rates are not allocated in conventional frequency bands ranging from $C$-band to $\mathrm{Ku}$-band. Such wide bandwidths are available only at higher frequency bands such as Ka-band $(20-30 \mathrm{GHz}), Q / V$-band $(40-50 \mathrm{GHz})$ or EHF-band $(20-45 \mathrm{GHz})$. For civilian applications, this trend is reinforced by the saturation of the conventional frequency bands ( $C$-band and Ku-band); hence, developing a new satellite system in these frequency bands may involve a lengthy co-ordination procedure in order to limit the interference caused by the new system to the existing ones (and vice versa).

For the next generation of satellite telecommunication systems, different types of system architecture are envisaged for multimedia applications [8] such as, for instance: broadband access star network with $\mathrm{Ku} / \mathrm{Ka}$-band transparent payloads and low-cost terminals for consumers and prosumers, broadband access mesh network with $\mathrm{Ku} / \mathrm{Ka}$-band regenerative payloads and VSAT terminals for prosumers and corporate, backbone core networks with $\mathrm{Ka} /$ $Q / V$-bands regenerative payloads for interconnection of gateways.

The main critical design issues for these systems, regarding the influence of the propagation channel, are the following:

- Design of system margin and availability (propagation channel): This is the conventional way to take into account the propagation effects in system design. However, at frequency bands higher than $20 \mathrm{GHz}$, rain attenuation is not the sole effect to be compensated. Also other effects such as gas and cloud attenuation as well as scintillation and possibly depolarization have to be considered.

- Implementation of FMTs (physical layer): These techniques aim at compensating in realtime strong propagation impairments that limit physical layer performance at $\mathrm{Ka}$ and $Q / V$ bands.

- Adaptive resource management (access layer): The purpose is to allocate dynamically the satellite resources with respect to propagation conditions in order to optimize the global system capacity.

\subsection{Propagation issues relevant for FMTs}

The implementation of FMTs leads to a specific design of adaptive systems, which have to react in real time to propagation conditions. FMTs are introduced into the system through the design of a control loop, which aims at mitigating a propagation event in real time. An efficient control 
loop should be able to track the signal variations, especially the slow component (attenuation), and to follow the envelope of fast fluctuations. The dynamics of the propagation channel is therefore a key element for system design at high-frequency bands and has to be taken into account directly in the definition of the FMT control loop. These dynamics of the propagation channel can be described in terms of fade slope and fade duration or of spectral characteristics. Fade slope and fade duration can help in optimizing internal parameters of the FMT control loop (response time of the loop, internal margins or guard time). Spectral characteristics are important in order to filter out quick variations of the signal due to scintillation.

Previous studies $[9,10]$ have demonstrated that the introduction of experimental propagation time series in the simulation of the performance of the physical layer allows FMT control loops to be optimized. Until now, experimental data have been used for this purpose by propagation experts, but they are not always available to system designers. In addition, experimental propagation data exist only for specific locations, frequencies and elevations. An alternative to real measured data for computer-based system simulations is to use flexible time-series synthesizers which can be adjusted to climatological characteristics as well as geometrical and radio-electrical link parameters. These models are not only able to calculate transfer functions, but also function as time-series synthesizers from which every kind of temporal characteristics can be extracted, such as the received signal evolution, transfer functions, spectral characteristics, and of course primary and secondary statistics.

\subsection{Requirements for channel models for the design of FMTs}

The aim of this research activity was to develop and validate propagation channel models in the form of time-series synthesizers; hence, the following system requirements are expressed for this kind of models only. These requirements have to specify the main characteristics and functionality to be fulfilled by the requested channel models, in order to be able to select the most appropriate ones. The specifications are expressed in terms of propagation effects to be simulated, input parameters, model outputs, global validity and flexibility and practical implementation.

The synthesizers developed and validated in this study allow time series of rain attenuation and scintillation to be generated. Both effects have to be taken into account because rain attenuation at frequencies higher than $10 \mathrm{GHz}$ is the main limitation for air interface performance, and scintillation adds noise to the FMT control loop parameters. Therefore, the capability of reproducing both effects is the main requirement. Also, clear sky attenuation effects (due to oxygen, water vapour and clouds) could be relevant, in particular at $40-50 \mathrm{GHz}$ or for low elevation links.

As far as input parameters are concerned, any possible configuration between an Earth station and a satellite has to be taken into account. First of all, the geometry of the link can be described by the positions of both the satellite and the Earth station. Furthermore, the radiowave characteristics must be considered in terms of frequency (between 10 and $50 \mathrm{GHz}$ ) and polarization. Moreover, the channel models should be able to take into account climatic conditions which are described by a number of radio-climatological parameters and can be obtained from all over the world by maps from ITU-R recommendations, maps generated from ECMWF data or maps from other meteorological offices.

Finally, the channel models should be able to extend to scenarios which have not been covered by measurements using statistical prediction models such as, for instance, Recommendations ITU-R P.618 for primary statistics or P.1623 for secondary statistics. 
In general, channel models must be capable of producing both short-term and long-term time series. Short-term time series correspond to the generation of event-on-demand that should be specified from its maximum attenuation and/or its total duration. This feature is important to test the behaviour of an FMT control loop with fade events that correspond to specific FMT activation thresholds (does the designed loop allow a specified attenuation to be actually mitigated?), to evaluate the switching rate between specific FMT modes or to assess the required signalling. Longterm time series are also useful to infer statistical distributions (availability, switching rate, throughput, etc.), for instance, on a monthly basis in order to analyze the impact of the seasonal variability on the optimization of control loop internal margins. It can be mentioned here that event-on-demand may also be selected with the appropriate algorithm from a long-term time series.

The possibility of changing sampling rate (e.g. between 0.1 and $100 \mathrm{~Hz}$ ) is another requirement for the channel models. In fact the type of system simulation determines the granularity of the propagation time series, e.g. if the performance of the control loop around a specific FMT activation threshold has to be determined, a rapid sampling rate is required, on the other hand, if the allocation of the satellite resources is tested with a network simulator, a lower sampling rate would be sufficient. The computational efficiency represents another requirement. It should be low in order to save computation time when synthesizing long-term time series.

\section{DESCRIPTION OF THE BASIC PRINCIPLES OF THE CHANNEL MODELS CONSIDERED IN THIS STUDY}

A wide review of channel models has been carried out by the same team [11] in the framework of the European action COST 280 ('Propagation impairment mitigation for millimetre-wave radio systems'). From this list of channel models, six models have been selected: spectral (SPL) model, two-sample model (TSM), second-order Markov chain (2MC) model, N-states Markov chain model, ITALSAT data-based model and synthetic storm technique (SST). This selection has been mainly based on the following criteria: detailed information available, good theoretical basis, maturity of approach, possibility of generalization to any part in the world.

\subsection{Spectral model (SPL)}

Relying on the theory described in $[12,13]$, this stochastic model partly aims at synthesizing rain attenuation time series [14,15] as well as scintillation time series [16]. Maseng and Bakken have made two main hypotheses concerning the rain attenuation process $A_{\text {rain }}$ :

- The long-term distribution of rain attenuation is log-normal, characterized by two parameters: the mean $m$ and the standard deviation $\sigma$ of its Neperian logarithm.

- Rain attenuation can be transformed into a first-order stationary Markov process using the nonlinear transformation:

$$
X=\frac{\ln A_{\text {rain }}-m}{\sigma}
$$

where $A_{\text {rain }}$ is rain attenuation in $\mathrm{dB}$.

Moreover, first-order stationary Markov processes are characterized by autocorrelation functions that follow the analytical expression:

$$
R_{X}(\tau)=\exp (-\beta|\tau|)
$$


Figure 1 shows the relationship between the white Gaussian noise generating source $n(t)$ and the instantaneous rain attenuation $A(t)$ in $\mathrm{dB}$ synthesized by the SPL model.

Three parameters are then necessary to generate rain attenuation time series with this model:

- The log-normal law parameters $m$ and $\sigma$, respectively, the mean and the standard deviation of the log-attenuation.

- The $\beta$ parameter introduced to describe the dynamics of rain attenuation.

The three parameters $m, \sigma$ and $\beta$ have to be determined on a long-term basis. In fact, they are dependent on the link considered and can be evaluated using time series of rain attenuation collected over long periods (months, years).

This channel model requires as inputs the geometrical and radiowave characteristics of the link, as well as the cut-off frequency of the rain filter, as shown in the flowchart given in Figure 2 [17]. An analysis tool has been developed to infer the latter from experimental time series.

\subsection{Two-sample model (TSM)}

This model predicts the probability distribution of rain attenuation a short time after a measured value, dependent on the values of two previous samples of rain attenuation [18]. This

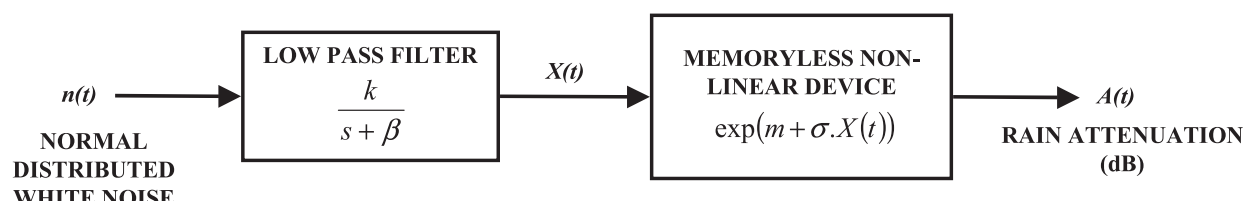

Figure 1. Principle of the spectral model.

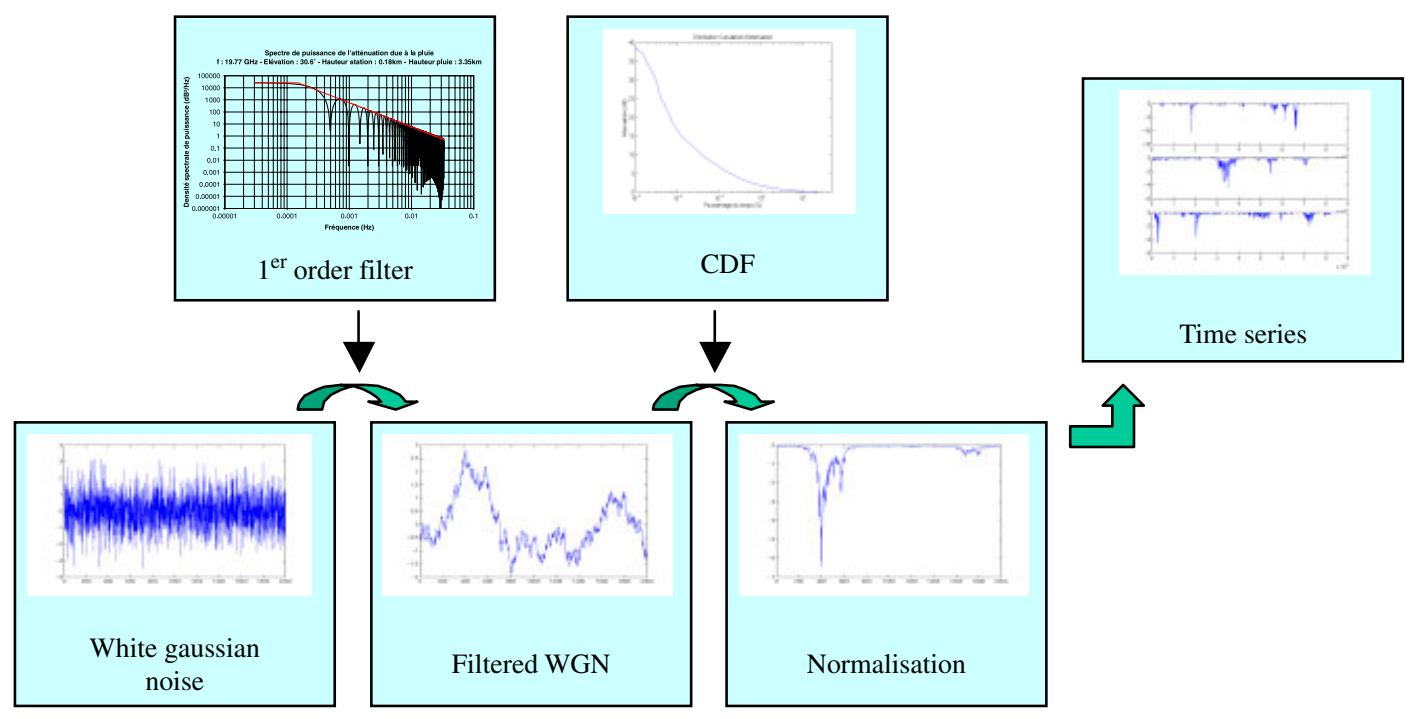

Figure 2. Spectral model flow chart. 
TSM enables adaptive fade countermeasure systems to anticipate the expected attenuation, and determine the required tracking speed. Also, with these results a model of rain attenuation as a Markov process can be derived, which can be used to develop data simulation procedures of rain attenuation.

The model derived is described as follows. The probability distribution $p(A)$ of attenuation $A$ at time $t+\Delta t$ is given by

$$
p(A)=\frac{m_{A}}{2 A \sigma_{A}} \operatorname{sech}\left(\frac{\pi m_{A} \ln \left(A / m_{A}\right)}{2 \sigma_{A}}\right)
$$

where $m_{A}$ and $\sigma_{A}$ are dependent on the two previous attenuation values $A_{0}$ at time $t$, and $A_{-1}$ at time $t-\Delta t$, as

$$
\begin{aligned}
m_{A} & =A_{0}\left(\frac{A_{0}}{A_{-1}}\right)^{\alpha_{2}} \\
\sigma_{A} & =A_{0} \sqrt{\beta_{2} \Delta t}+A_{0} \gamma_{2}\left(1-\mathrm{e}^{-\left|\ln \left(A_{0} / A_{-1}\right)\right|}\right)
\end{aligned}
$$

Two separate sets of parameters have been identified for a time step of 10 and $1 \mathrm{~s}$, respectively:

$$
\begin{aligned}
& \Delta t=10 \mathrm{~s}, \quad \Delta t=1 \mathrm{~s} \\
& \alpha_{2}=0.40, \quad \alpha_{2}=0.77 \\
& \beta_{2}=2.0 \times 10^{-4} \mathrm{~s}^{-1}, \quad \beta_{2}=2.9 \times 10^{-5} \mathrm{~s}^{-1} \\
& \gamma_{2}=0.43, \quad \gamma_{2}=0.25
\end{aligned}
$$

This channel model requires as inputs the maximum attenuation or the maximum duration of the rain fade event, as well as a set of coefficients $\alpha_{2}, \beta_{2}$ and $\gamma_{2}$, corresponding to the considered sampling rate $(1$ or $0.1 \mathrm{~Hz})$. These coefficients have been derived from a large data set of OLYMPUS and ITALSAT propagation measurements [19] and are assumed to be independent of frequency, elevation and climate for frequencies ranging between 10 and $50 \mathrm{GHz}$, for elevations ranging between 27 and $30^{\circ}$ and for maritime European climates. An analysis tool has been developed to infer these three parameters from experimental time series.

\subsection{Second-order Markov chain model (2MC)}

This model is based on a $2 \mathrm{MC}$ [4]. It can be run in either a discrete-valued or a continuousvalued version. The continuous-valued version represents an improvement of the discrete one, since it provides not only higher resolution but also allows for a slightly less complex implementation of the model (see Figure 3).

The transition probabilities of the $2 \mathrm{MC}$ are modelled as Gaussian random variables in such a way that a single Gaussian random variable fully describes all $N$ transitions from a particular state to all possible other states. Note that $N$ represents the number of attenuation values in the range from $0 \mathrm{~dB}$ to typically $30 \mathrm{~dB}$. A quantization of $1 \mathrm{~dB}$ yields 31 states, a finer quantization of $0.1 \mathrm{~dB}$ results in 301 states. The complete Markov chain would require the knowledge of a total of $N^{2}$ Gaussian distributions. However, by pooling transitions in the manner described in Figure 3 , a total of only $3 N$ Gaussian distributions have to be taken into account, i.e. $3 N$ mean values and $3 N$ standard deviations have to be determined. 


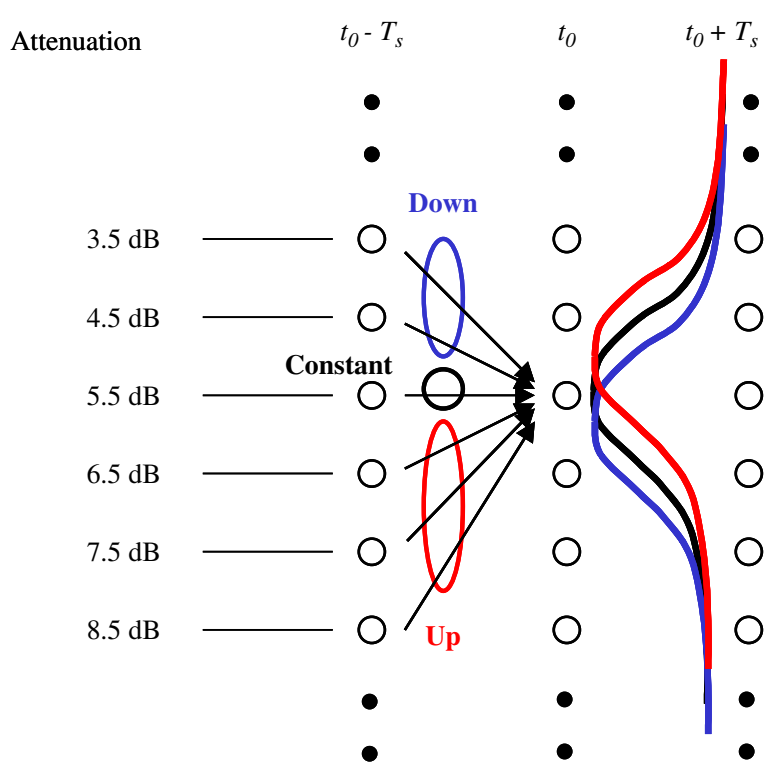

Figure 3. Second-order Markov chain with $N$ states and a $1 \mathrm{~dB}$ quantization; three Gaussian distributions per state reflect the history at time instant $t_{0}-T_{s}$.

The set of these $3 N$ mean values and $3 N$ standard deviations represents the specific channel model parameters (SCMPs) of the 2MC model. Figure 4 gives an example for the SCMPs. Note that in this figure the quantization of the attenuation levels is $1 \mathrm{~dB}$.

This channel model requires as input the set of $3 N$ mean values and standard deviations. This set has to be determined from measurements. It is available for two European locations as outcome of the ITALSAT experiments. An analysis tool has been developed to infer these sets also from other experimental time series. The $2 \mathrm{MC}$ model inherently provides continuous synthetic time series including fade and inter-fade periods and allows the consideration of diurnal and seasonal variations but does not provide fades on demand.

\section{4. $N+2$-States Markov chain model (MKV)}

The $N+2$-states Markov chain (MKV) model aims at synthesizing time series of rain attenuation only [20]. The model is divided into three parts. The first part is referred to as macroscopic model and provides a time series consisting of two possible states: 'rain' and 'no rain'. An example of such a time series is shown in Figure 5. The second part of the model is the so-called microscopic model. Its task is to fill the boxes, in other terms to generate attenuation time series according to the value of the states obtained from the macroscopic model. The microscopic model provides the short-term dynamic behaviour of rain attenuation (through the fade slope distribution). Figure 6 shows an example of a time series of duration $D$ generated by the microscopic model. The last part of the MKV model consists in combining the two time series obtained from the previous parts in order to generate complete long-term time series. 


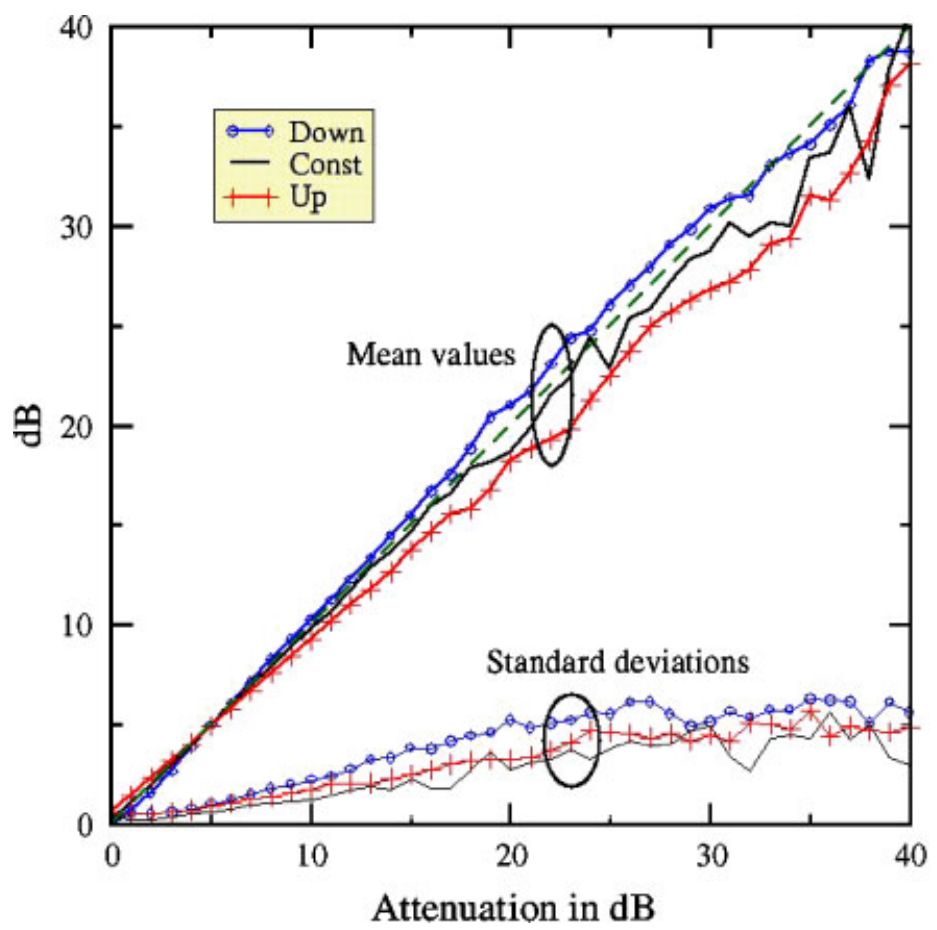

Figure 4. Example of the SCMPs for the second-order Markov chain model.

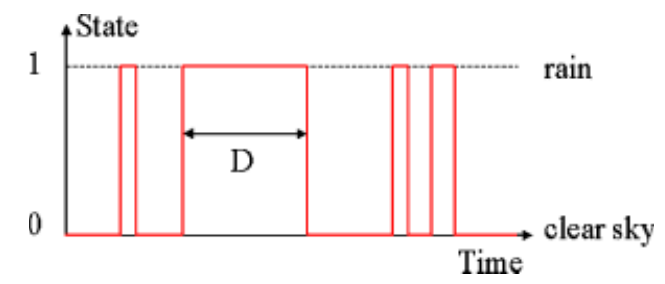

Figure 5. Example of 2-states time series of rainy periods of time.

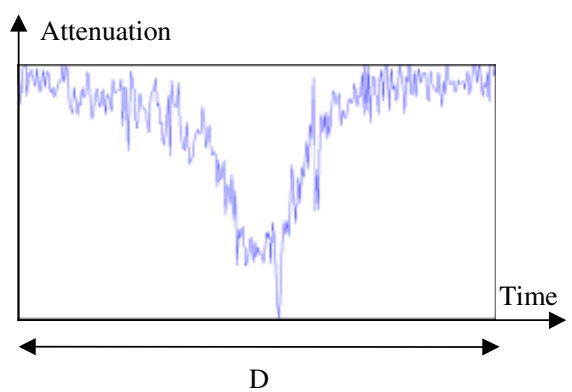

Figure 6. Example of $N$-states time series. 
This rain attenuation time-series synthesizer relying on $N$-state Markov chains has been developed in order to offer a great flexibility to the user in terms of time and attenuation granularity and in terms of the opportunity to control some characteristics of a propagation event like its maximum attenuation and if possible, its total duration.

The transition probabilities of the $N$-states Markov chain model are based on Recommendation ITU-R P.1623 which predicts fade slopes for different sampling rates and cut-off frequencies. The input data requested to run the model are geometrical configuration (satellite and Earth station locations), radiowave characteristics (frequency and polarization) and the climatological parameters being given by ITU-R radio-meteorological maps.

\subsection{ITALSAT data-based model (IDB)}

This synthesizer of rain attenuation time series [21] is based on a large set of experimental rain attenuation time series collected at Ka- and $V$-bands between 1994 and 2000 in the framework of the ITALSAT propagation experiment, ended in January 2001 [22]. This database of experimental rain attenuation time series is large enough to reproduce the main features of the phenomenon.

The database consists of event files containing rain attenuation values in $\mathrm{dB}$ and sampled every second for the three beacons at $18.7,39.6$ and $49.5 \mathrm{GHz}$ (from now on referred to as 19,40 and $50 \mathrm{GHz}$ ). Attenuation was then cleaned of system biases by using a set of radiometers. Then, scintillation was filtered out (low-pass Butterworth filter with a cut-off frequency of $0.025 \mathrm{~Hz}$ ) and rain attenuation events were extracted by using specific thresholds to constitute the database. An example of a rain event at the three frequencies is shown in Figure 7.

The principle of the time-series synthesizer consists in extracting rain attenuation events and in dividing these rain attenuation events in classes according to their peak attenuation and then scaled in frequency, polarization, elevation, rain height, etc. to take into account the conditions

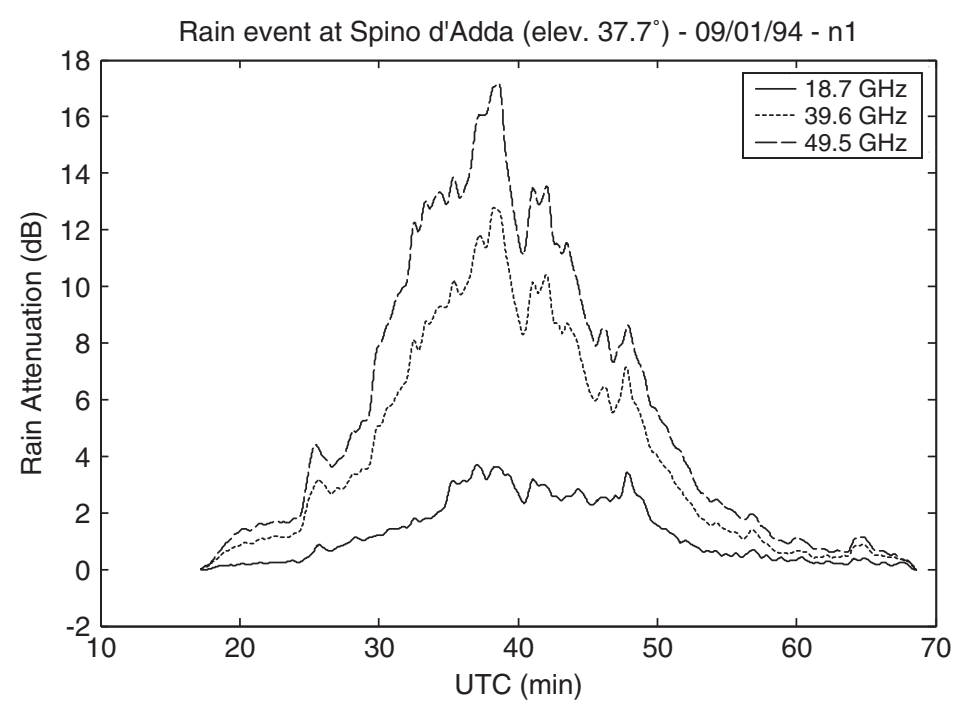

Figure 7. Example of ITALSAT rain attenuation events. 
existing in a different location. Finally, a particular procedure determines the weights of the different classes of rain attenuation events so as to reproduce the cumulative distribution function of rain attenuation as measured or predicted for a generic link in a generic location. The block diagram of the synthesizer is shown in Figure 8.

This channel model requires as inputs the geometrical and radiowave characteristics of the link, as well as the cut-off frequency of the rain filter. A database containing the time series of measured attenuation for several rain events with different typologies and intensities is also required. A database of more than 2500 events is available from ITALSAT measurements at Spino d'Adda. In order to limit the dimension of the database and to facilitate both portability and accessibility of the database, a total of 500 events representing different months and years has been selected. A classification of these events with respect to their peak attenuation level is required. Typically 20-30 classes representing the peak attenuation in $\mathrm{dB}$ have to be distinguished to obtain best results.

\subsection{Synthetic storm technique (SST)}

Starting from the rain rate time series of rain storm, collected at a site with a rain gauge, the SST can generate rain attenuation time series at any frequency and polarization, for any slant path above about $20^{\circ}$, at this site [23]. A synthetic storm is obtained by converting a rain rate time series, recorded at a point by a rain gauge (a robust and economic device) and averaged over $1 \mathrm{~min}$ (or less, as experimented with the ITALSAT database collected at Spino d'Adda), to a rain rate space series along a line, by using an estimate of the storm translation speed $v$ to transform time to distance, through a convolution integral. It has been shown that the statistical properties of rain attenuation derived from a large sample of rain rate time series closely agree with those of the actual measured rain attenuation, and that the first-order statistics are significantly insensitive to the value of $v$ (fade duration and slope statistics being mainly determined by the movement of the storms). Rain attenuation time series can be simulated by using the average value of $v$, obtainable from measurements of wind speed at about the 700mbar level, often available from meteorological services.

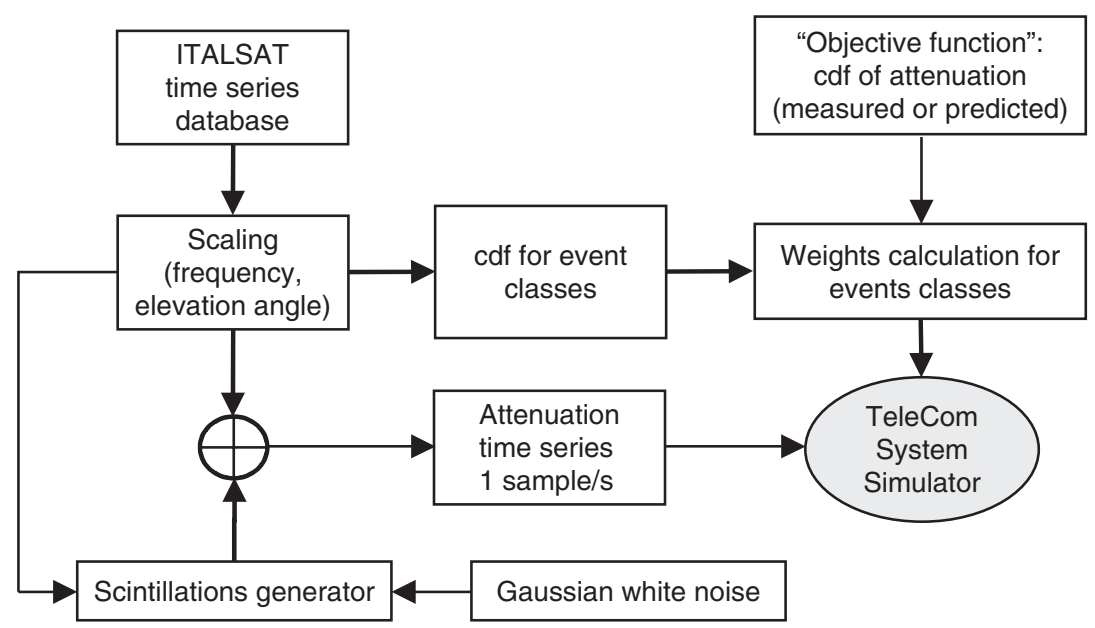

Figure 8. Block diagram of the ITALSAT data-based time-series synthesizer. 
The vertical structure of rain is modelled with two layers of precipitation of different depths. Starting from ground, there is rain (hydrometeors in the form of raindrops, water temperature of $20^{\circ} \mathrm{C}$, layer A) followed by a melting layer (layer B), i.e. melting hydrometeors at $0^{\circ} \mathrm{C}$. The vertical rain rate $R(\mathrm{~mm} / \mathrm{h})$ in the lower layer is assumed to be uniform and given by the rain rate measured at ground, i.e. by the rain gauge. By assuming simple physical hypotheses, calculations show that also the vertical precipitation rate in the melting layer, termed 'apparent rain rate', can be supposed to be uniform and given by $3.134 R$. The height of the precipitation (rain and melting layer) above sea level is assumed to be the ITU-R $0^{\circ} \mathrm{C}$ isotherm height above sea level given, for a site in the Northern hemisphere at latitude $\phi \geqslant 23^{\circ}$, by $5-0.075 \cdot(\phi-$ 23) $\mathrm{km}$, and the depth of the melting layer is $0.4 \mathrm{~km}$, regardless of the latitude. As far as rain attenuation is concerned, the two layers (A and B) describe, on the average and effectively, all rain events made up of the two main types of precipitation, i.e. stratiform and convective. Rain attenuation, $A(\mathrm{~dB})$, is calculated as a line integral of the specific rain attenuation $Y=k R^{\alpha}$ $(\mathrm{dB} / \mathrm{km})$, according to the values of rain rate found in the rain rate space series obtained with the SST.

This channel model requires as inputs the geometrical and radiowave characteristics of the link, as well as a database containing time series of measured rain rate (1-min integration time or less) for the site in question.

\section{COMPARISON WITH EXPERIMENTAL DATA}

\subsection{Methodologies for comparison with experimental data}

4.1.1. Methodology for testing. Two methodologies have been followed to test the above described time-series synthesizers against experimental data. A 'long-term testing' activity is presented in the present paper in order to validate the long-term behaviour of the synthesized channel as compared with real ones. An 'event-based' testing activity, not described in the present article, has also been developed to assess the detailed short-term behaviour during a fading event; it is the object of companion papers [24, 25].

The experimental data used to perform the tests are the following:

- Seven years of recordings from the ITALSAT campaign in Spino d'Adda, Italy (called 'SPI' in the following) at 19 and $50 \mathrm{GHz}$ between 1994 and 2000.

- Four years of recordings from the ITALSAT campaign in Oberpfaffenhofen, Germany (called 'OBE' in the following) at $40 \mathrm{GHz}$ between 1994 and 1997.

- Two years of recordings collected during the OLYMPUS campaign simultaneously in Lessive (Belgium, called 'LSV' in the following) at $20 \mathrm{GHz}$ and in Louvain-la-Neuve (Belgium, called 'LLN' in the following) at $30 \mathrm{GHz}$ in 1990 and 1992.

As the considered time-series synthesizers generate attenuation only, scintillation is filtered out from the experimental time series, using a fifth-order Butterworth filter; a cut-off frequency of $0.025 \mathrm{~Hz}$ is used, as recommended by OPEX [26].

The long-term testing activity methodology consists in generating 10 data sets with each time-series synthesizer, each of these 10 data sets corresponding to a total duration of 1 year. The motivation to generate a number of 10 data sets is to get an idea of the yearly dispersion of 
the results obtained with each of the time-series synthesizers. The testing activity is performed in terms of first-order statistics (rain attenuation distributions) and second-order statistics (fade slope and fade duration distributions). Because the selected second-order statistics (fade slope and fade duration) describe the dynamical properties of the propagation channel which are very important for the design of FMTs a lower ranking will be assigned to first-order statistics testing (attenuation distributions) in the evaluation of performances. The testing variables used for rain attenuation and fade duration are the ones given in Recommendation ITU-R P. 311, whereas the testing variable for fade slope is given by [27]:

$$
\text { err }=\ln \left(\frac{P_{\text {predicted }}}{P_{\text {measured }}}\right)
$$

4.1.2. Time-series generation procedures. Not each model could produce the same type of time series as the other channel models. Differences are due to the fact that

- some of them (e.g. $2 \mathrm{MC}$ model) produces continuous time series of total attenuation including clear sky periods,

- another group of channel models (MKV, SST, SPL) produce continuous time series of excess attenuation including inter-event periods (that is with periods where there is no rain attenuation),

- the last group of channel models (TSM, $N$-states Markov chain and ITALSAT-based models) produce only rain attenuation events with no information on the inter-event period.

It therefore appears necessary to define a methodology to generate comparable time series and to appropriately compare their statistics against those of experimental data.

As different types of time series can be generated, in the following, the analysis will be concentrated on comparisons of rain attenuation event collections, which is the common output of all channel models. To be able to compare objectively the synthesized rain attenuation time series, the same cumulative duration of rain attenuation events will be generated. The generation of the same number of attenuation events has been rejected, because it would result both in a very large number of small rain attenuation events and in different cumulative durations.

Our approach was to consider only a certain amount of cumulative duration in order to make sure that it will correspond to rain periods for each experimental location. This cumulative duration is fixed to $1 \%$ of an average year (that is on a part where rain attenuation is the dominant effect). Furthermore, we have decided to take into account only parts of generated or experimental fade events with attenuation higher than the attenuation exceeded for $1 \%$ of the time given, respectively, by $A_{20 \mathrm{GHz}}(1 \%)=0.6 \mathrm{~dB}$ for Lessive, $A_{30 \mathrm{GHz}}(1 \%)=1.6 \mathrm{~dB}$ for Louvain-la-Neuve, $A_{19} \mathrm{GHz}(1 \%)=1.5 \mathrm{~dB}$ and $A_{50 \mathrm{GHz}}(1 \%)=5.5 \mathrm{~dB}$ for Spino d'Adda, and finally $A_{40 \mathrm{GHz}}(1 \%)=2.9 \mathrm{~dB}$ for Oberpfaffenhofen.

\subsection{Results in terms of rain attenuation distributions}

The testing activity on rain attenuation is performed for the channel models with respect to the OLYMPUS data collected in Louvain-la-Neuve and Lessive (Belgium, near Brussels) and with respect to the ITALSAT data collected in Spino d'Adda (Italy, near Milan). Results obtained with Recommendation ITU-R P.618 are also given as reference in terms of prediction accuracy. 
Figure 9 shows the results in terms of complementary cumulative distribution function (CDF) of attenuation obtained from synthesized and experimental time series at around $20 \mathrm{GHz}$ (in Lessive and Spino d'Adda), where the CDFs of rain attenuation obtained from each channel model correspond to the whole period of the 10 generated data sets. The relative error between the CDF of synthesized attenuation (over 10 years) and each single year experimental CDF is shown in the lower row of Figure 9.

The results obtained with the time-series synthesizers adapted to the Lessive scenario show a very good agreement for the ITALSAT data-based model and the SPL model down to $0.02 \%$. $2 \mathrm{MC}$ model and TSM approximate less well the experimental CDF, with differences that can reach $2 \mathrm{~dB}$ in Lessive for $0.02 \%$ of the time.

Obviously, the ITALSAT data-based model at both frequencies shows the best agreement with the measurements at Spino d'Adda, which represents its reference data set. The $2 \mathrm{MC}$
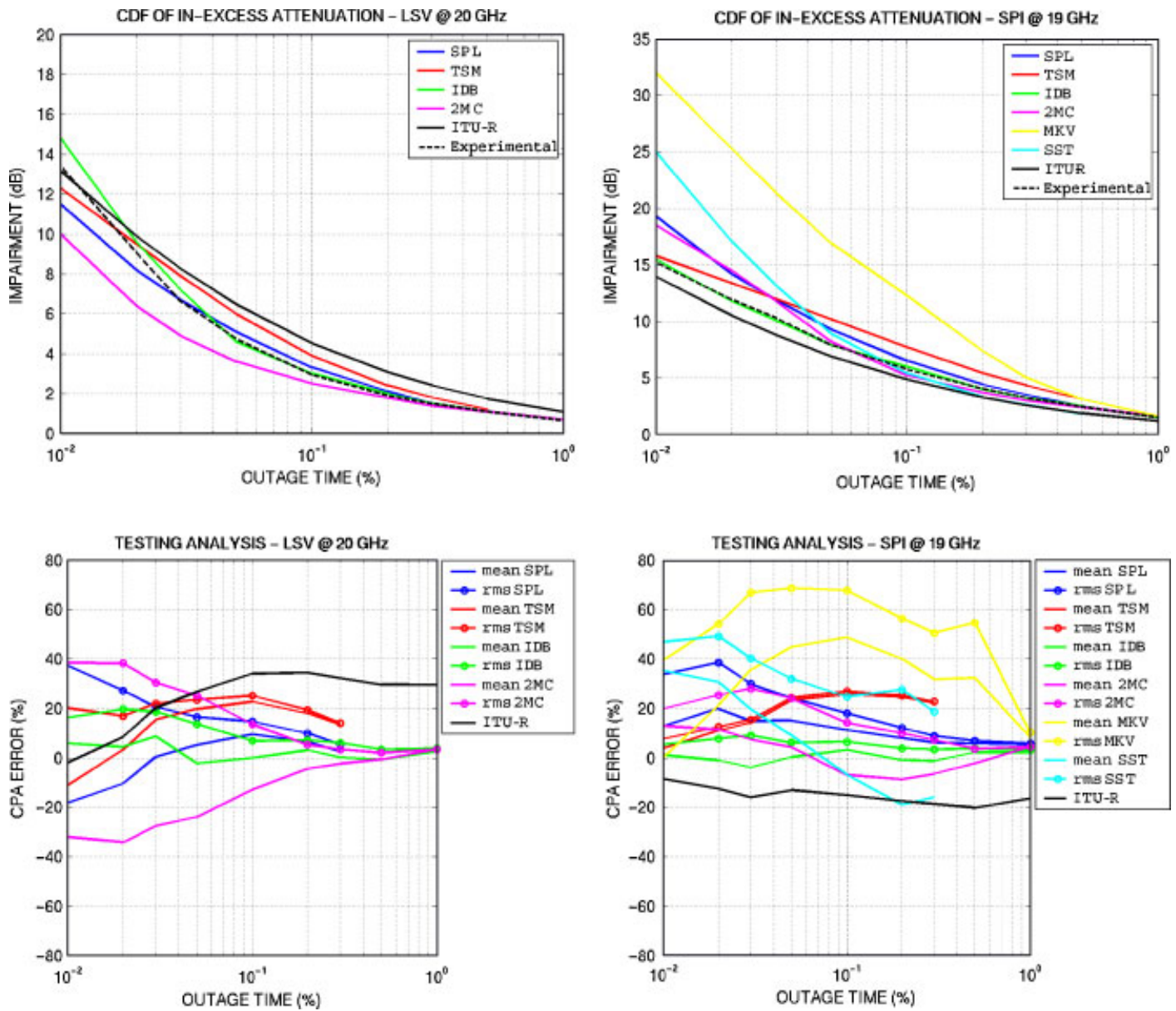

Figure 9. Comparison between CDF of rain attenuation obtained from synthesized data and CDF of rain attenuation obtained from experimental data in Lessive (upper left) and Spino d'Adda (upper right); the corresponding error statistics are given in Lessive (lower left) and in Spino d'Adda (lower right). 
model, the SPL model and the SST show a very good agreement down to $0.05 \%$, the TSM giving slightly worse agreement. Results obtained with the $N$-states Markov chain are not satisfactory with a strong overestimation of the experimental CDF. Concerning the SST, the overestimation of the experimental curve is logical as this technique relies on the conversion to attenuation of the concurrent rain rate time series, whose rain gauge availability is higher than the beacon one [28].

A similar analysis conducted at $30 \mathrm{GHz}$ in Louvain-la-Neuve, at $40 \mathrm{GHz}$ in Oberpfaffenhofen and at $50 \mathrm{GHz}$ in Spino d'Adda is shown in Figure 10. Here again, good results are obtained with all the represented time-series synthesizers.

The results of the dispersion analysis are presented in Figure 11 for Spino d'Adda only, which gives the variability obtained with each time-series synthesizer, presented with the driest (1998) and the wettest (1999) ITALSAT CDFs.
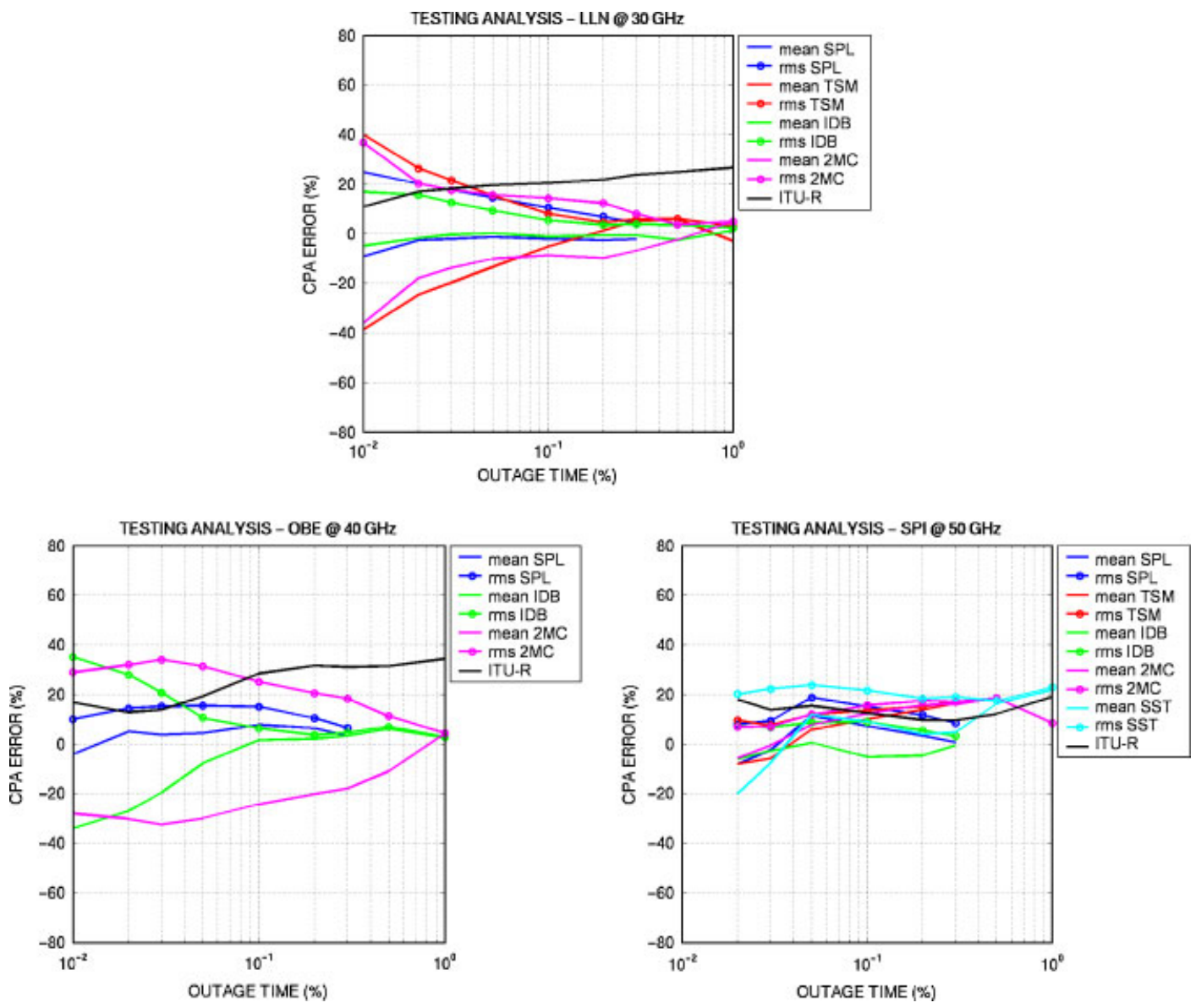

Figure 10. Error statistics of rain attenuation obtained from synthesized and experimental data at $30 \mathrm{GHz}$ in Louvain-la-Neuve (upper) at $40 \mathrm{GHz}$ in Oberpfaffenhofen (lower left) and at $50 \mathrm{GHz}$ in Spino d'Adda (lower right). 

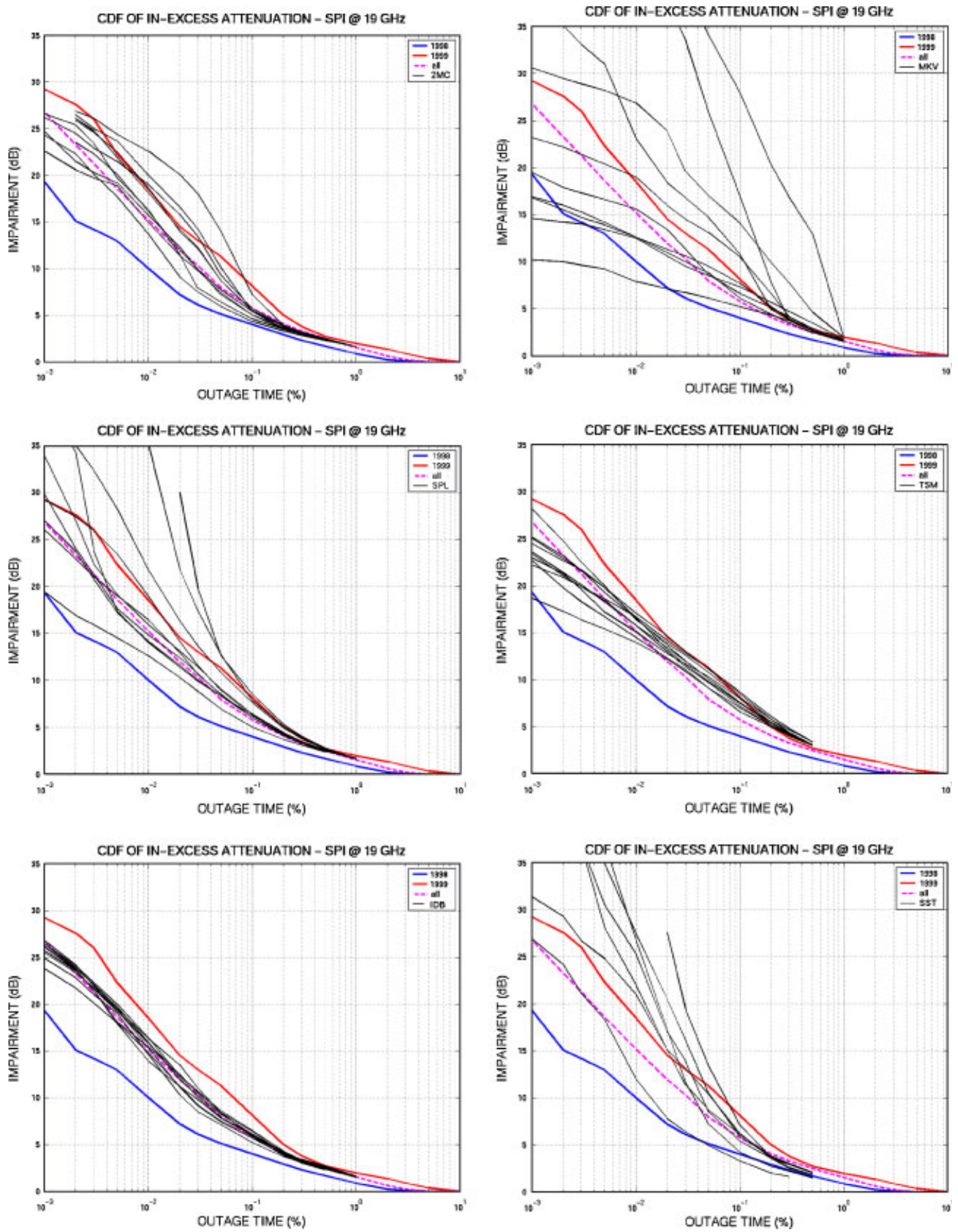

Figure 11. Dispersion of the attenuation CDF obtained at $19 \mathrm{GHz}$ with the channel models with respect to Spino d'Adda measured variability. 
The analysis of the results displayed in Figure 11 at $19 \mathrm{GHz}$ shows with respect to experimental data:

- the ITALSAT data-based model (low middle) shows little or no yearly dispersion,

- the TSM (lower left hand) and the 2MC model (upper left hand) show a lower but comparable yearly dispersion data,

- the SPL model (upper right hand) and the SST (lower right hand) exhibit higher but comparable yearly dispersion data,

- the $N$-states Markov chain model (upper middle) exhibits the highest yearly dispersion.

Apart from these specific results, the yearly dispersion is surprisingly similar for all the timeseries synthesizers.

The main conclusions of the testing activity on attenuation distributions are the following. Very good results (better than with Recommendation ITU-R P.618) are obtained with 2MC, SPL, TSM and ITALSAT data-based models for all frequencies and locations (especially for SPL and ITALSAT data-based models). The dispersion of the results obtained from the generation of 10 independent years of time series is similar to the one observed on the experimental data for $2 \mathrm{MC}$ model, SPL model and TSM as well as for the SST.

However, as already stated, the testing activity related to attenuation distributions is assumed to be less relevant than the testing of dynamical properties and represents more a validation rather than an accuracy assessment procedure.

\subsection{Results in terms of distributions of attenuation duration}

The testing activity on fade duration is performed for the channel models with respect to the whole multiple-year distributions measured in Lessive, Louvain-la-Neuve and Spino d'Adda. Results obtained with Recommendation ITU-R P.1623 are also given as reference in terms of prediction accuracy.

In this section, attenuation duration refers to the duration of attenuation, obtained after filtering of the synthesized and experimental time series with a raised square cosine filter used with a cut-off frequency of $0.02 \mathrm{~Hz}$ as recommended during the OPEX [26].

The fade duration distributions as defined in Recommendation ITU-R P.1623 are normalized with respect to the minimum duration or in other words with respect to the time between adjacent samples (for all distributions, probability corresponding to a $1 \mathrm{~s}$ attenuation duration threshold is set equal to 1). The distribution of short fades is not used in the present testing analysis. In the following, attenuation duration distributions are produced for each location considering a minimum duration of:

- $1 \mathrm{~s}$ for $N$-states Markov chain, SPL, TSM and ITALSAT data-based models as well as SST applied to Spino d'Adda, Louvain-la-Neuve and Lessive locations,

- $60 \mathrm{~s}$ for all models and all locations.

The first series of comparisons between distributions of the relative number of fades (probability of occurrence of fades of duration $d$ longer than $D$ in seconds, given that the attenuation $a$ is greater than $A$ in $\mathrm{dB}$ ) obtained from synthesized and experimental time series are presented in Figure 12 for the data collected at around $20 \mathrm{GHz}$ (Lessive and Spino d'Adda). The distributions of the relative number of fades obtained from each channel model correspond to the whole period of the 10 generated data sets. These ' 10 years' distributions are then 


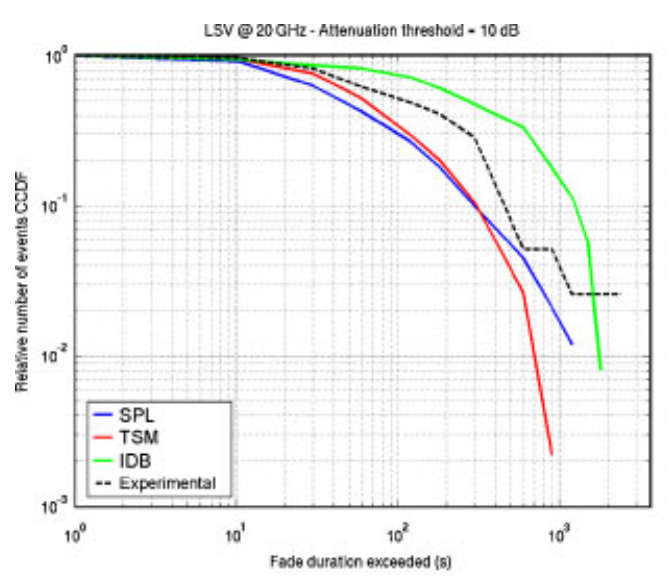

LSV@ $20 \mathrm{OHz}$

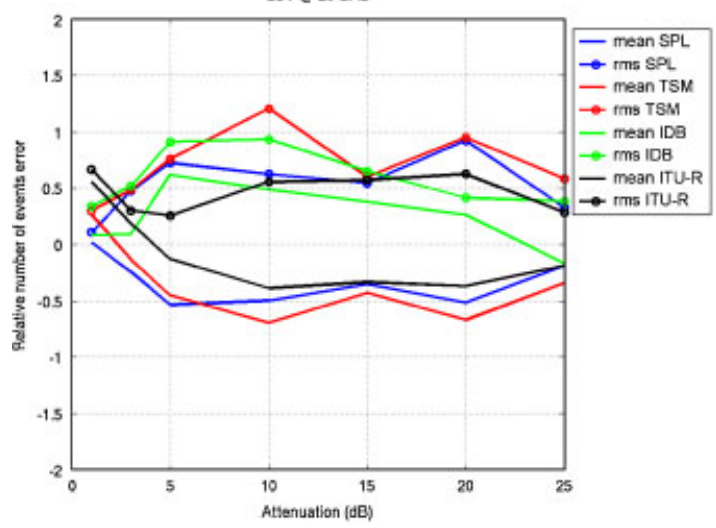

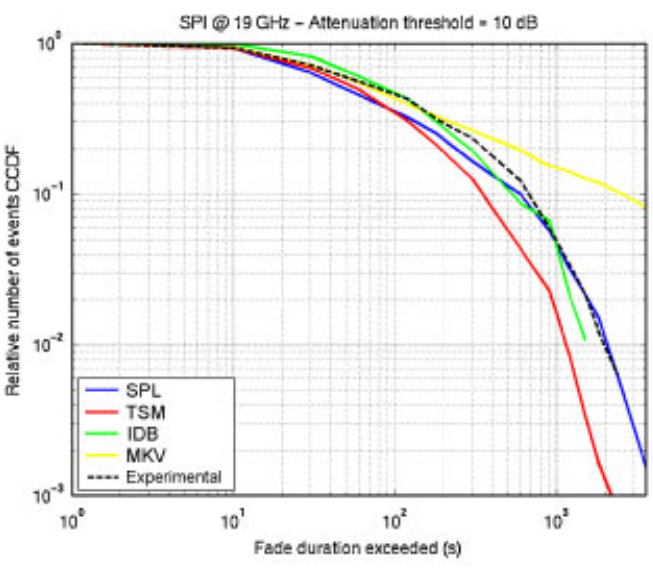

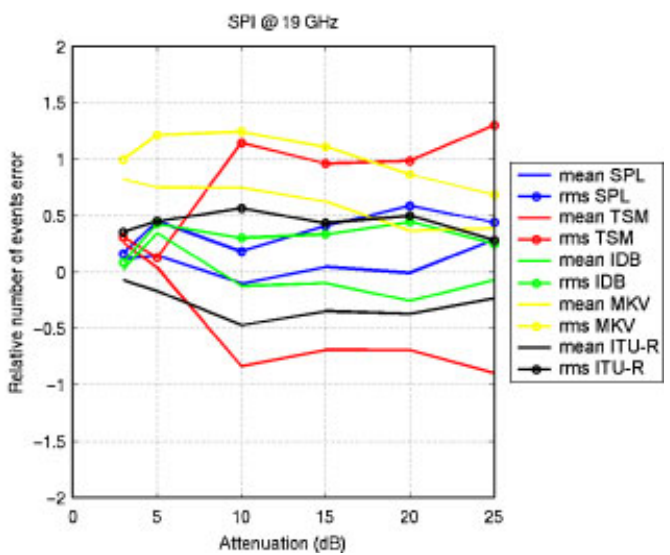

Figure 12. Comparison between distributions of the relative number of fades obtained from synthesized data and of relative number of fades obtained from experimental data in Lessive (left) and Spino d'Adda (right); the corresponding error statistics are given in Lessive (lower left) and Spino d'Adda (lower right).

compared with the experimental distribution calculated over the whole multiple-year data collected in Lessive and Spino d'Adda and to the distributions of the relative number of fades predicted with Recommendation ITU-R P.1623. Error statistics are then calculated over the different thresholds.

It can be seen from Figure 12 that SPL, TSM and ITALSAT data-based models perform very well, which is not the case of the $N$-states Markov chain model in Spino d'Adda. Results obtained with SST cannot be objectively compared with the other models for 1-s attenuation duration, as this technique proved to be applicable essentially for $60 \mathrm{~s}$ duration (see instead Figure 13) [23].

In order to assess the performances of 2MC and SST models, the analysis is repeated using durations longer than $60 \mathrm{~s}$. Consequently, all distributions are set equal to 1 for a 60 -s attenuation duration threshold (in the previous case, all distributions equalled to 1 for a 1-s attenuation duration threshold) to allow comparisons with 2MC and SST models (see Figure 13).

The following conclusions can be derived from the analysis of Figure 13: the $2 \mathrm{MC}$ model and the SPL model exhibit fair performances for all locations, with an error similar to 

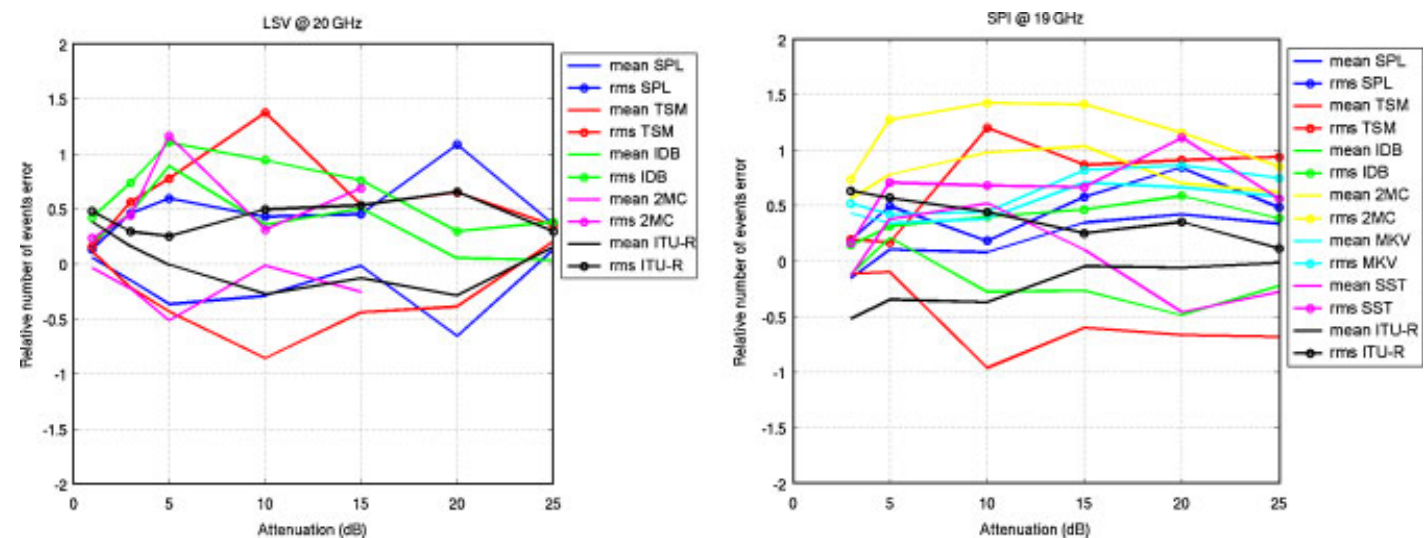

Figure 13. Variation of the 60-s attenuation duration testing variable for the relative number of fades with respect to attenuation threshold obtained from synthesized data and experimental data in Lessive (left) and Spino d'Adda (right).

Recommendation ITU-R P.1623, the other models giving higher RMS error; here, the $N$-states Markov chain model does appear to provide acceptable results, with particular regards for attenuation events longer than $30 \mathrm{~min}$.

A similar analysis is presented in Figure 14 which gives the error statistics calculated between simulations performed with the time-series synthesizers and experimental $60 \mathrm{~s}$ fade duration distributions calculated at $30 \mathrm{GHz}$ in Louvain-la-Neuve, at $40 \mathrm{GHz}$ in Oberpfaffenhofen and at $50 \mathrm{GHz}$ in Spino d'Adda. Here, the SPL model performs better than Recommendation ITU-R P.1623 for all these three locations at these three frequencies.

Another analysis has been performed on the total fraction of time (due to fades of duration $d$ longer than $D$ in seconds, given that the attenuation $a$ is greater than $A$ in $\mathrm{dB}$ ), which showed the following conclusions: concerning the accuracy of models with respect to experimental statistics of the total fraction of $1 \mathrm{~s}$ fade duration intervals, the best accuracy is obtained with the ITALSAT data-based model and the SPL model for all frequencies and locations; concerning the accuracy of models with respect to experimental statistics of the total fraction of $60 \mathrm{~s}$ fades duration intervals, the best accuracy is obtained with the SPL model followed by the ITALSAT data-based model, the 2MC model and the TSM for all frequencies and locations.

\subsection{Results in terms of distributions of attenuation slope}

In a first step, attenuation slopes are calculated between two consecutive samples (i.e. over $1 \mathrm{~s}$ ). In this case, this is not strictly the physical attenuation slope which is investigated, since the time series has been filtered with a cut-off frequency of $0.025 \mathrm{~Hz}$ (time constant around $40 \mathrm{~s}$ ), but instead distortion to attenuation given by the filter. However, this situation is relevant for an FMT control loop, where a 1-s monitored signal can be filtered to remove scintillation, and where the fade slope can be used to improve short-term prediction [10].

A first series of comparisons between CDFs of $1 \mathrm{~s}$ attenuation slope obtained from synthesized and experimental time series collected at around $20 \mathrm{GHz}$ (Lessive and Spino d'Adda) are presented in Figure 15, for an attenuation threshold of $10 \mathrm{~dB}$. In this figure the 

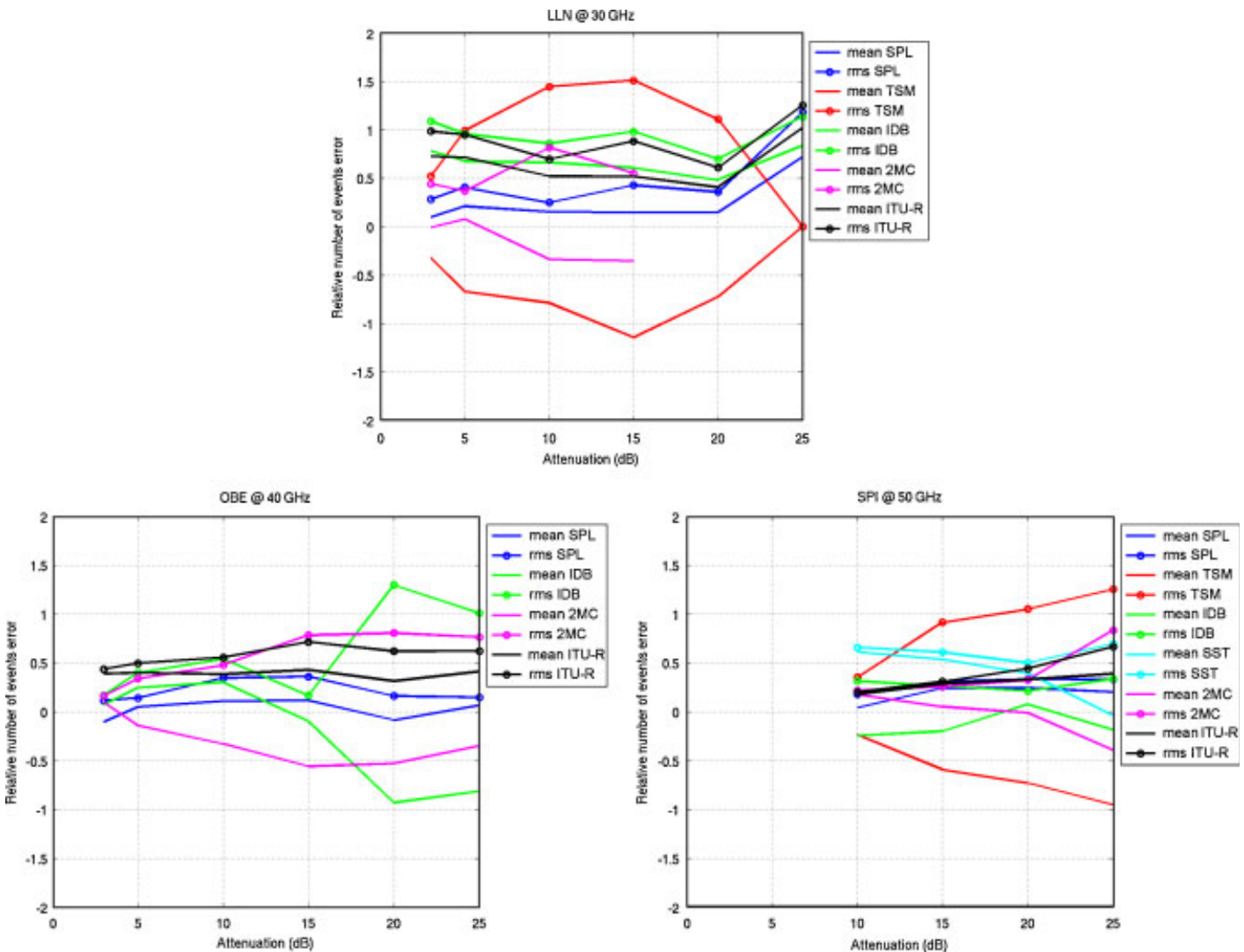

Figure 14. Error statistics on the distributions of the relative number of fades obtained from $60 \mathrm{~s}$ synthesized data and of relative number of fades obtained from $60 \mathrm{~s}$ experimental data at $30 \mathrm{GHz}$ in Louvain-la-Neuve (upper), at $40 \mathrm{GHz}$ in Oberpfaffenhofen (lower left) and at $50 \mathrm{GHz}$ in Spino d'Adda (lower right).

CDFs of $1 \mathrm{~s}$ attenuation slope obtained from each channel model correspond to the whole period of the 10 generated data sets. These ' 10 years' distributions are then compared with the experimental CDF calculated over the whole multiple-year distributions measured in Lessive and Spino d'Adda and to the CDF of $1 \mathrm{~s}$ attenuation slope predicted with Recommendation ITU-R P.1623. As for attenuation duration, only models allowing time series to be generated at $1 \mathrm{~s}$ sampling rate are considered in this testing analysis.

For this $10 \mathrm{~dB}$ attenuation threshold, the shape of the experimental curve is well approximated by the SPL, the TSM and the ITALSAT data-based models. Results obtained with the $N$-states Markov chain model are relatively far from the experimental curve. More generally, for low percentages of time (lower than 1\%), the time-series synthesizers seem to underestimate the attenuation slope.

When attenuation slopes are calculated over $1 \mathrm{~min}$ instead of $1 \mathrm{~s}$ (see Figure 16 to allow comparisons with the 2MC model and with the SST model), first of all a decrease of the attenuation slope values can be observed, which do not exceed $0.3 \mathrm{~dB} / \mathrm{s}$ instead of a maximum 

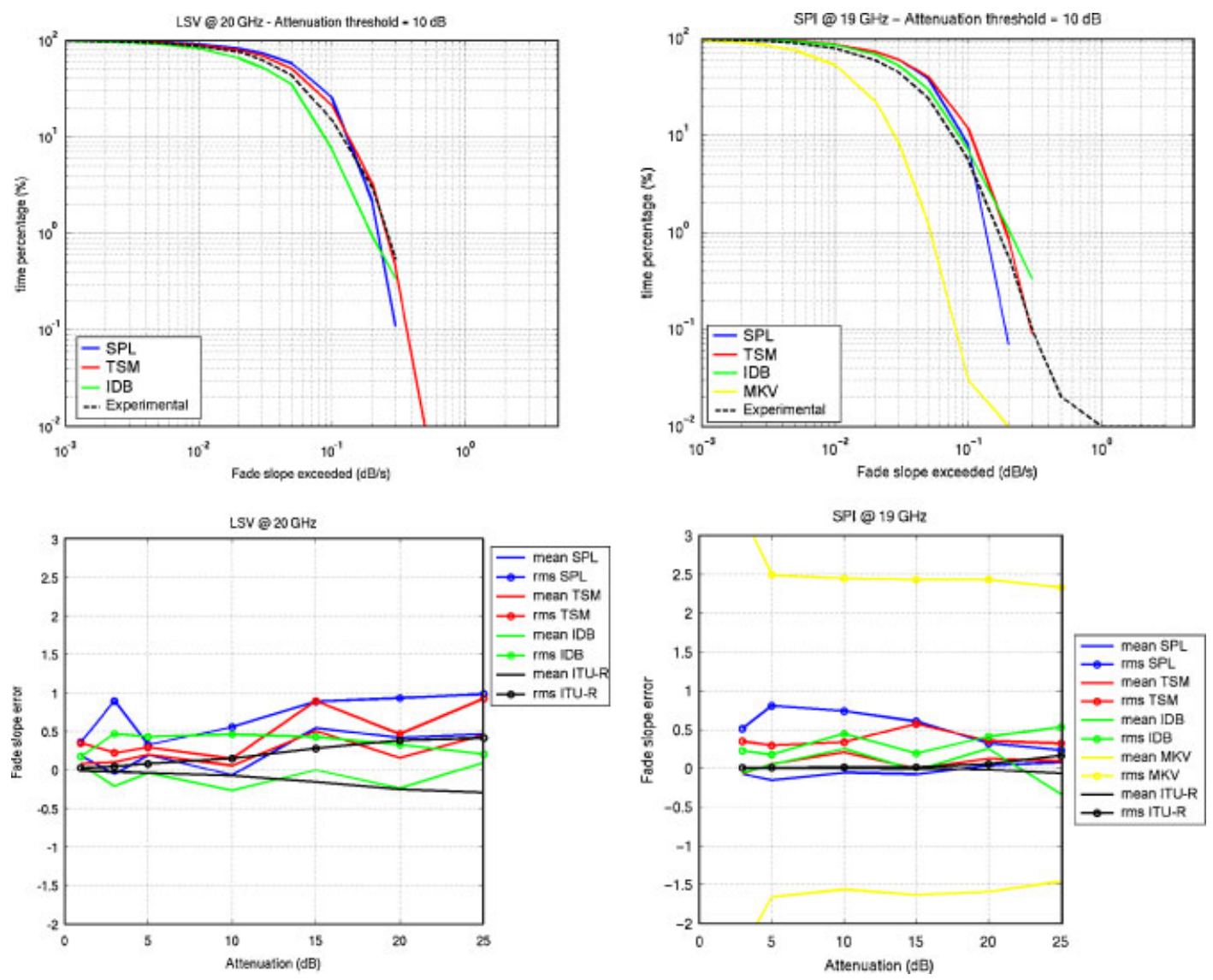

Figure 15. Comparison between CDF of $1 \mathrm{~s}$ attenuation slope obtained from synthesized data and of $1 \mathrm{~s}$ attenuation slope obtained from experimental data in Lessive (upper left) and Spino d'Adda (upper right); the corresponding error statistics are given in Lessive (lower left) and Spino d'Adda (lower right).

value of $1 \mathrm{~dB} / \mathrm{s}$ for $1 \mathrm{~s}$ attenuation slopes. Secondly, Recommendation ITU-R P.1623 performs better than previously and also better than the time-series synthesizers. It can be noticed that here, this is really the time derivative of the attenuation which is considered.

Afterwards, the accuracy of the channel models is degraded for the SPL and the $N$-states Markov chain models, stable for the TSM and improved for the ITALSAT data-based model and for the SST. Generally, the best results are obtained by the ITALSAT data-based model, followed by $2 \mathrm{MC}$ model, TSM and the SST and then by the SPL model.

Finally, a similar analysis is conducted as shown in Figure 17 which presents the errors calculated between synthesized and experimental $60 \mathrm{~s}$ attenuation slope distributions obtained at $30 \mathrm{GHz}$ in Louvain-le-Neuve and at $50 \mathrm{GHz}$ in Spino d'Adda. Here again, Recommendation ITU-R P.1623 performs better than the time-series synthesizers, especially at 40 and $50 \mathrm{GHz}$, where other effects, not taken into account in the time-series synthesizers, would have to be considered. 

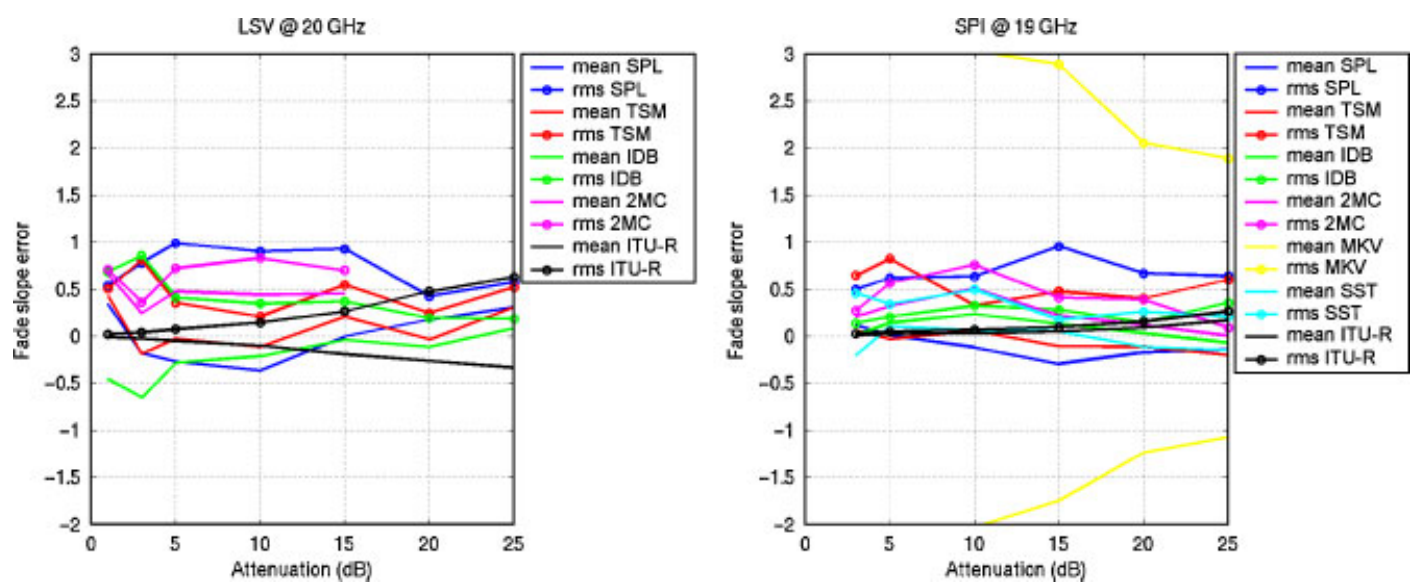

Figure 16. Error statistics of $60 \mathrm{~s}$ attenuation slope obtained from synthesized data and experimental data in Lessive (left) and Spino d'Adda (right).

\section{CONCLUSION}

In this paper, the context of multimedia satellite telecommunication systems has been outlined focussing on the necessary adaptivity of these systems (using interference and fade mitigation techniques, adaptive resource management). Then the needs of propagation channel models have been presented, expressed in terms of time-series generation.

Afterwards, a collection of six time-series synthesizers able to answer to these needs has been reviewed and their main principles have been described: spectral model, two-samples model, second-order Markov chain model, $N$-states Markov chain model, ITALSAT data-based model and synthetic storm technique. Improvements of the implementation of these models have been recently achieved.

Finally, some comparisons of synthesized time series against measured data collected during the OLYMPUS and the ITALSAT propagation experiments have been presented, which have shown good agreement between the long-term distributions obtained from the synthesis of time series and the experimental distributions.

This study has allowed the following main progress on channel models to be achieved:

- a set of channel models has been developed with consolidated theoretical basis,

- a set of analysis tools has been developed for retrieving internal parameters of the channel models from experimental measurements,

- a methodology has been designed for long-term testing of channel models,

- a specific methodology has been built for short-term or event-based testing of channel models,

- comparison results of channel models performances have been obtained with respect to an experimental database for first-order, second-order and fade event characteristics.

Some improvements can be brought on these time-series synthesizers, such as the possibility to synthesize events-on-demand, that is with the control of the maximum attenuation and of the total duration of each generated attenuation event. On the other hand, the validation of these 

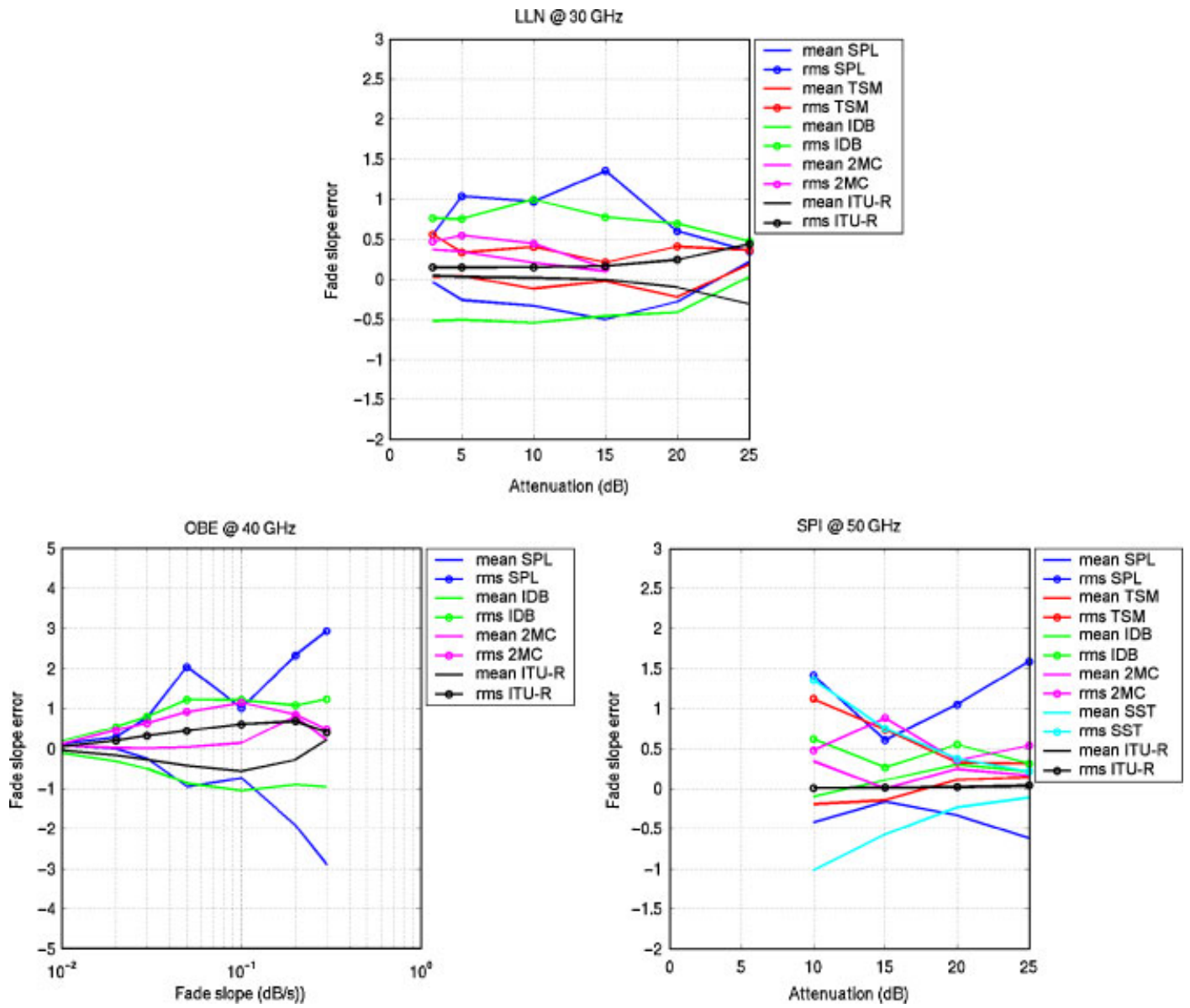

Figure 17. Error statistics of attenuation slope obtained from $60 \mathrm{~s}$ synthesized data and $60 \mathrm{~s}$ experimental data at $30 \mathrm{GHz}$ in Louvain-la-Neuve (upper), at $40 \mathrm{GHz}$ in Oberpfaffenhofen (lower left) and at $50 \mathrm{GHz}$ in Spino d'Adda (lower right).

time-series synthesizers can be extended, particularly for other link elevations and other climates (especially for tropical and equatorial areas), thanks to the availability of automatic analysis tools allowing the internal parameters of the models to be retrieved from experimental time series. Finally, other effects than rain attenuation have to be modelled in these frequency bands, such as oxygen, water vapour and cloud attenuation as well as scintillation, which need to be implemented in the presented models.

This study has been carried out:

- in the framework of the ESA contract no. 16865/03/NL/EC 'Development of propagation models for telecommunication satellite systems', with contributions from Frederic LACOSTE (previously TéSA as ONERA-CNES PhD student, now with CNES) and from Max VAN DE KAMP (previously ONERA, now University of Bath), 
- in the framework of the European action COST 280 ('Propagation impairment mitigation for millimetre-wave radio systems'),

- in the framework of the European Network of Excellence SatNEx.

\section{ACKNOWLEDGEMENTS}

The authors want to thank Danielle VANHOENACKER from Université Catholique de Louvain-la-Neuve to have provided the OLYMPUS data, and ASI (Italian Space Agency) for the use of the ITALSAT data.

\section{REFERENCES}

1. Arbesser-Rastburg B et al. Radiowave propagation modelling for new satcom services at Ku-band and above. COST 255 Final Report, ESA Publications Division, SP-1252, March 2002.

2. Castanet L, Lemorton J, Bousquet M. Fade mitigation techniques for New SatCom services at Ku-band and above: a review. Fourth Ka-band Utilization Conference, Venice, November 1998.

3. Castanet L, Mertens D, Audoire B, Bousquet M, Czarnecki M, Page A, Vander Vorst A, Vasseur H. Ka-band videoconference VSAT system. COST 255 Final Report, Chapter 6.2, ESA Publications Division, SP-1252, March 2002.

4. Fiebig U-C. Channel modelling for Ka-band and above. Proceedings of the Personal Indoor Mobile Radio Conference, PIMRC'99, Osaka, Japan, 1999.

5. Shin SK, Lee SI, Kim YS, Kim JM. A dynamic rain attenuation modelling technique for satellite communication link. Proceedings of the International Conference on Telecommunications, Cheju, Korea, 1999; 2:33-37.

6. Audoire B, Castanet L, Christophe F, Gremont B. Ka band attenuation time series generator and comparison with OLYMPUS data. AIAA-ICSSC-19 Conference, Toulouse, France, April 2001.

7. Filip M, Martellucci A, Willis M, Bousquet M. COST 280: propagation impairment mitigation for millimetre wave radio systems. Proceedings of the ICAP 2003, March-April 2003, University of Exeter: U.K. See also http:// www.cost280.rl.ac.uk/

8. De Gaudenzi R, Del Rio-Herrero O, Arbesser-Rastburg B, Coromina F, Sabbadini M, Hollreiser M, Bellini M. Satellite broadband R\&D system concepts \& technological directions. ESA Workshop on Techniques and Technologies for Future Satellite Broadband Systems, Noordwijk, The Netherlands, December 2001.

9. Castanet L, Mertens D, Bousquet M. Simulation of the performance of a Ka-band VSAT videoconferencing system with uplink power control and data rate reduction to mitigate atmospheric propagation effects. International Journal of Satellite of Communications 2002; 20:231-249.

10. Bolea-Alamanac A, Castanet L, Leconte K, Bousquet M. FMT control loop performance assessment on a point-topoint oriented satellite broadband system for multimedia applications. Tenth Ka-band Utilization Conference, Vicenza, Italy, October 2004.

11. Fiebig U-C, Castanet L, Lemorton J, Matricciani E, Perez-Fontan F, Riva C, Watson R. Propagation channel modelling. COST 272-280 International Workshop on Satellite Communications from Fade Mitigation to Service Provision, Noordwijk, The Netherlands, May 2003.

12. Maseng T, Bakken PM. A stochastic model for rain attenuation. IEEE Transactions on Communications 1981; COM-29(5):660-669.

13. Gremont B, Filip M. Spatio-temporal rain attenuation model for application to fade mitigation techniques. IEEE Transactions on Antennas and Propagation 2004; 52(5):1245-1256.

14. Lacoste F, Bousquet M, Castanet L, Cornet F, Lemorton J. Improvement of the ONERA-CNES rain attenuation time series synthesiser and validation of the dynamic characteristics of the generated fade events. Space Communications 2005; 20:45-59.

15. Lacoste F, Bousquet M, Castanet L, Cornet F, Lemorton J. A new channel model to synthesise rain attenuation time series. IEEE Transactions on Antennas and Propagation, submitted.

16. Lacoste F, Millerioux JP, Castanet L, Riva C. Generation of time series of scintillation combined with rain attenuation. European Conference on Propagation and Systems, Brest, March 2005.

17. Castanet L, Cornet F. Simulator of the dynamic behaviour of the propagation channel for Ka-band satcom systems. CLIMDIFF 2003, Fortaleza, Brazil, 17-19 November 2003.

18. Van de Kamp MMJL. Rain attenuation as a Markov process, using two samples. Post-doctorate Thesis, Document ONERA RT 1/06733.04 DEMR, Toulouse, France, March 2003.

19. Lemorton J, Castanet L, Lacoste F, Van de Kamp MMJL, Riva C, Matricciani E, Fiebig U-C. Development of propagation models for telecommunication satellite systems. ESA study no. 16865/03/NL/EC, ONERA Final Report RF 4/07757/DEMR, September 2004. 
20. Castanet L, Deloues T, Lemorton J. Methodology to simulate long-term propagation time series from the identification of attenuation periods filled with synthesized events. COST 272-280 International Workshop on Satellite Communications from Fade Mitigation to Service Provision, Noordwijk, The Netherlands, May 2003.

21. Valbonesi L. A large database of ITALSAT attenuation data for tropospheric channel simulations. First COST 280 Workshop, doc. PM3-006, Malvern, U.K., 1-3 July 2002.

22. Riva C. Seasonal and diurnal variations of total attenuation measured with the ITALSAT satellite at Spino d'Adda at 18.7, 39.6 and 49.5 GHz. International Journal of Satellite Communications and Networking 2004; 22(4):449-476.

23. Matricciani E. Physical-mathematical model of the dynamics of rain attenuation based on rain rate time series and two layer vertical structure of precipitation. Radio Science 1996; 31:281-295.

24. Lacoste F, Bousquet M, Castanet L, Cornet F, Lemorton J. Methodology of validation of time series synthesiser for the Ka-band satellite propagation channel. AIAA/ICSSC'2004 Conference, Monterrey, U.S.A., May 2004.

25. Lacoste F, Bousquet M, Castanet L, Cornet F, Lemorton J. Event-based testing analysis of rain attenuation time series synthesizers for the ka-band satellite propagation channel. CLIMDIFF Conference, Cleveland, OH, U.S.A., September 2005.

26. OPEX: Reference book on attenuation measurements and prediction. Second Workshop of the on Attenuation Distributions Propagation Experimenters (OPEX), Doc. ESA-ESTEC-WPP-083, Noordwijk, The Netherlands, November 1994.

27. Van de Kamp MMJL. Test of fade duration and fade slope models. CLIMDIFF, Fortaleza, Brazil, November 2003.

28. Matricciani E, Riva C. The search for the most reliable long-term rain attenuation pdf of a slant path and the impact on prediction models. IEEE Transactions on Antennas and Propagation 2005; 53:3075-3079.

\section{AUTHORS’ BIOGRAPHIES}

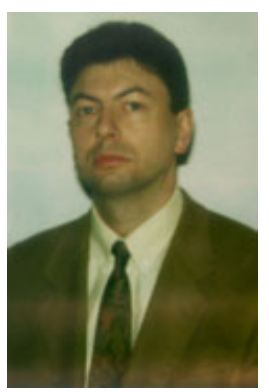

Joël Lemorton received in 1983 the Diploma of Engineering in Electronics from ENSEEIHT, Toulouse, and in 1986 the PhD degree in Electronics from SupAero Toulouse. Since 1984 he has been working in the field of phased arrays antennas, remote sensing systems and radiowave propagation at the Microwave Department of ONERA (the French Aerospace Lab). Since 1997, Dr Lemorton has been responsible for the 'Antennas and Radiowave Propagation' Research Unit of the Electromagnetism and Radar Department of ONERA. His current research interests are mainly focused on Ka and EHF bands Earth-Space telecommunications. His team is active on innovative antenna design and technology, and on radiowave propagation studies (terrestrial and Earth-Space propagation) for radar, navigation and telecommunications systems. These studies concern propagation measurements campaign (CELESTE, STENTOR, HB6, GSAT4), statistical prediction models, space and time dependent channel models, physical models and systems studies (Fade Mitigation Techniques), and are conducted for ESA, CNES and EU. Since 1991, he has been involved as a French expert in COST 235, COST 255 and COST 280 European projects, and in the European Network of Excellence 'SatNEx'. Dr Lemorton is also teaching Electromagnetism and Remote sensing and has been supervisor for several $\mathrm{PhD}$ students. He is the author of several papers and a lot of presentations in international conferences. He is a member of URSI Commission F.

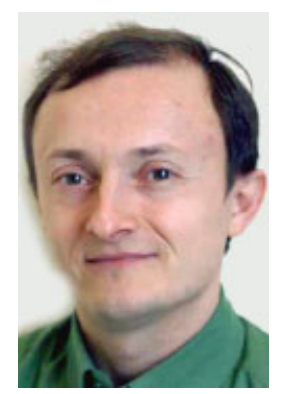

Laurent Castanet was born in France in 1967. He received the BS degree in Microwave Engineering from TELECOM BRETAGNE in 1991 and the MS Degree in Space Communications Systems from TELECOM PARIS in 1992. He received the PhD Degree from SupAero in 2001. He works as a research engineer in the radiowave propagation field at ONERA Toulouse. His main research interests are Earth-Space propagation and Fade Mitigation Techniques (FMT). He was a French expert in the COST 255 and COST 280 European projects, in which he worked on the modelling of the space-time variability of the propagation channel and on the design and optimisation of adaptive FMT. At the moment, Laurent Castanet is involved in the European Network of Excellence 'SatNEx', in which he chairs the propagation Joint Activity, and participates to the physical layer and access Joint Activities. He is also a French delegate in ITU-R Study Group 3 which deals with radiowave propagation. 


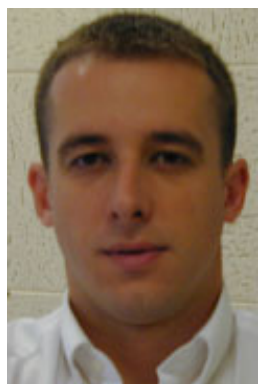

Frédéric Lacoste received the MSc Degree from ENSICA, Toulouse, France in 2002 and the PhD degree in Propagation and Signal Processing in 2005 from SupAero, Toulouse, France. Since 2005, he is working as a research engineer in the French Space Agency (CNES) in the field of RF propagation and signal processing within projects preparing future Satellite Communications and Radionavigation systems. His current work interests include tropospheric propagation effects on Earth-Space links at Ka-band and above and environment propagation effects at L-, S- and C-bands for hybrid systems including both satellite and terrestrial transmitters.

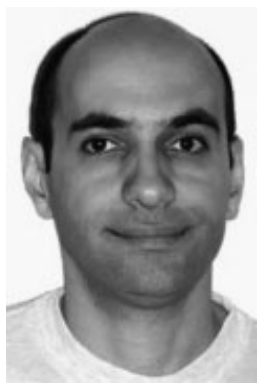

Carlo Riva (M'97) was born in Italy in 1965. He received the laurea degree (cum laude) in Electronic Engineering and the Doctorate degree in Electronic and Communication Engineering in the field of millimeter-wave propagation through the atmosphere from the Politecnico di Milano, Milan, Italy, in 1990 and 1995, respectively. In 1992, he visited the European Space Research and Technology Centre, Noordwijk, The Netherlands, working on scintillation. In 1993, he visited the Universite Catholique de Louvain, Louvain-la-Neuve, Belgium, doing research on the separation of turbulence and rain effects on satellite communication links. $\mathrm{He}$ has been participating in the COST 255 and COST 280 international project on propagation and telecommunications. He is currently with Politecnico di Milano as an Associate Professor of Electromagnetic Fields.

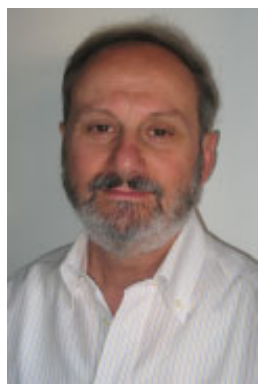

Emilio Matricciani was born in Italy in 1952. After serving in the Army, he received the Laurea degree in Electronics Engineering from Politecnico di Milano, Milan, Italy, in 1978. He joined Politecnico di Milano in 1978 as a recipient of a research scholarship in Satellite Communications, and in 1981, he became an Assistant Professor of Electrical Communications. In 1987, he joined the Università di Padova, Padua, Italy, as an Associate Professor of Microwaves. Since 1991, he is with Politecnico di Milano as an Associate Professor of Telecommunications. In the year 2001, he qualified as a Full Professor. He teaches Telecommunications to undergraduate students and Engineering of Satellite Communication Systems to graduate students. In addition to these institutional activities, he also teaches the fundamental aspects of scientific writing (theses, reports, papers) to undergraduate, graduate, master and doctorate students at Politecnico di Milano. His research activity started in the 1970s when he was involved in the radio propagation experiments conducted with the Italian satellite Sirio in the 1970s in the 12-14 and $18 \mathrm{GHz}$ bands, and continued in the 1980s and 1990s when he was in the team who planned and conducted the experiments with Italsat in the 20-30 and $40-50 \mathrm{GHz}$ bands. His actual research interests include satellite communications for fixed and mobile systems, deep-space communications, radio wave propagation, history of physics and telecommunications.

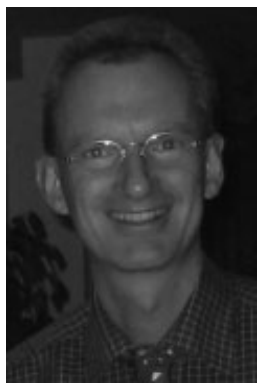

Uwe-Carsten Fiebig was born in Augsburg, Germany, in 1962. He studied Electrical Engineering at the Technical University of Munich (TUM), Germany, and spent the last year of his studies at Ecole Supérieure en Electrotechnique et Electronique in Paris, France. In 1987 he received the Dipl.-Ing. degree from TUM and in 1993 the Dr.-Ing. degree from University of Kaiserslautern. Mr Fiebig joined the Institute of Communications and Navigation at DLR (German Aerospace Research), Oberpfaffenhofen, Germany, in 1988. In 1995 and 1996 he was guest scientist at the Communications Research Center, Ottawa, Canada, and at the Yokohama National University, Japan, respectively. Since $1998 \mathrm{Mr}$ Fiebig is Head of the Department Communications Systems which carries out research in satellite navigation, aeronautical communications and mobile radio. His current research interests include channel modelling for satellite communication and satellite navigation systems. He teaches satellite communications and navigation at University of Ulm, Germany, and University of Linz, Austria. 
M. J. L. van de Kamp was born in Driebergen, The Netherlands, in 1963. He received the MSc degree in Electrical Engineering in 1989, and the PhD degree in Telecommunications in 1999, both from Eindhoven University of Technology ('EUT'), The Netherlands.

He has been working as a research scientist in various radiowave propagation research projects: in 1990-1994 at EUT, in 1995-1997 at Helsinki University of Technology, Finland, in 1997-1999 again at EUT, in 2000-2003 at Onera, Toulouse, France, in 2003-2005 as an external research fellow for the European Space Agency ('ESA'), and since 2006 at the University of Bath, U.K. His activities in these projects have been studies of rain and ice depolarisation, tropospheric and ionospheric scintillation, and dynamic aspects of rain attenuation.

During his research jobs, he has been involved in international cooperational satellite propagation research projects of ESA: the Olympus Propagation Experiment ('OPEX') in 1991-1994, COST 255 in 1995-1999, and COST 280 in 2001-2005.

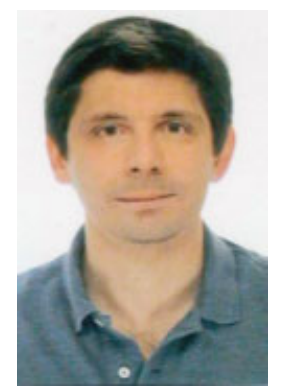

Antonio Martellucci received the Laurea degree in Electrical Engineering and the $\mathrm{PhD}$ degree in Applied Electromagnetics from University of Rome 'La Sapienza', Rome, Italy in 1987 and 1992 respectively. In 1988 he joined as optical engineer for Selenia (now part of Finmeccanica) group where he worked on the development of optical active systems. From 1989 to 2000 he worked at the Fondazione Ugo Bordoni 'Radio communication Systems Division', Rome, Italy as a researcher on atmospheric propagation effects for terrestrial and spatial radio communication systems. During this period he participated the OLYMPUS and ITALSAT propagation experiments (through the ESA OPEX and Italian CEPIT working groups) for the measurement and modelling of the atmospheric attenuation and depolarization at $\mathrm{Ka}, \mathrm{Q}$ and $\mathrm{V}$ frequency bands. He also took part to European COST 210 and 255 projects and various ESA projects on rain scatter, clear air propagation modelling and climatological databases. Since 2001 he joined the European Space Agency, ESA-ESTEC, The Netherlands, Directorate of Technical and Quality Management, as a radiowave propagation engineer where he is currently involved in ESA Telecommunication (ARTES), Navigation (Galileo), Earth Observation (ENVISAT) and Science (Bepi-Colombo) Programmes. At ESA he is currently involved on models for multimedia SatCom systems, including fade mitigation techniques, modelling and characterization of tropospheric effects for navigation systems and development of ground propagation equipment.

Dr Martellucci was the recipient of the Young Scientist Award of XXV URSI General Assembly in 1996. He has been the general editor of the EU COST 255 and since 2001 he was member of the COST 280 Management committee. He is also member of the ESA delegation at ITU-R SG3. He is author of more than 90 publications in books, International Journals and conference proceedings. 